\title{
Cup coproducts in Hopf cyclic cohomology
}

\author{
Mohammad Hassanzadeh • Masoud Khalkhali
}

Received: 19 March 2012 / Accepted: 19 April 2013 / Published online: 5 December 2013

(C) Tbilisi Centre for Mathematical Sciences 2013

\begin{abstract}
We define cup coproducts for Hopf cyclic cohomology of Hopf algebras and for its dual theory. We show that for universal enveloping algebras and group algebras our coproduct recovers the standard coproducts on Lie algebra homology and group homology, respectively.
\end{abstract}

Keywords Cyclic cohomology $\cdot$ Hopf algebras $\cdot$ Noncommutative geometry

Mathematics Subject Classification (2010) $\quad$ 19D55 - 16T05 - 11M55

\section{Introduction}

In this paper we define cup coproducts for Hopf cyclic cohomology of Hopf algebras by establishing Künneth formulas for Hopf cyclic cohomology. Cup product for cyclic cohomology of algebras was first defined by Connes in [4]. Künneth formulas and (co)products for cyclic theory of algebras has been established by several authors $[1,2$, $8,16,17,19$ ] (cf. also Loday's book [24] and references therein for a full account). Ever since the discovery of Hopf cyclic cohomology by Connes and Moscovici and with computations carried on in $[6,7]$, it was clear that this theory and its dual counterpart [20] is a noncommutative analogue of Lie algebra homology and group homology. This of course poses a natural question: to what extent cup products in Lie algebra and group

Communicated by Jonathan Rosenberg.

M. Hassanzadeh $(\varangle) \cdot$ M. Khalkhali

Department of Mathematics, University of Western Ontario, London, ON, Canada

e-mail: mhassanz@uwo.ca

M. Khalkhali

e-mail:masoud@uwo.ca 
cohomology can be extended to Hopf cyclic cohomology. This question is answered in this paper. We use the theory of cyclic modules and the definition of Hopf cyclic cohomology (with coefficients) in terms of cyclic modules $[6,13]$ to define our coproducts in a natural way via Eilenberg-Zilber isomorphisms for cyclic modules. This gives us an external coproduct. By specifying to cocommutative Hopf algebras we then define cup coproducts for Hopf cyclic (co)homology of cocommutative Hopf algebras.

This paper is organized as follows. In Sects. 2 and 3 we recall the EilenbergZilber isomorphism and Künneth formula for cyclic homology. In Sect. 4 we recall the definition of Hopf cyclic cohomology with coefficients in an stable anti-YetterDrinfeld module and work out the Eilenberg-Zilber isomorphism and Künneth formula in the context of Hopf cyclic cohomology. In Sect. 5 we define external coproducts for Hopf cyclic cohomology and in Sect. 6 by restricting to cocommutative Hopf algebras we obtain internal coproducts on Hopf cyclic cohomology. It is known that when restricted to universal enveloping algebras, Hopf cyclic cohomology reduces to Lie algebra homology [6]. In Sect. 6 we show that under these isomorphisms our coproduct coincides with the coproduct in Lie algebra homology. In Sect. 7 we look at the dual Hopf cyclic homology as defined in [20] and define a coproduct on it. It is shown in [20] that when restricted to group algebras, Hopf cyclic homology is isomorphic to group homology. In this section we check that under this isomorphism our coproduct coincides with coproduct in group homology.

It should be mentioned that the term cup product is also used for a different type of operation in Hopf cyclic theory. Thus the cup products defined and studied in [11,13$15,22,25]$ involve an action of a Hopf algebra on a (co)algebra and is a pairing between Hopf cyclic cohomology of the given (co)algebra and Hopf cyclic cohomology of the acting Hopf algebra. This operation is in fact an extension of Connes-Moscovicvi's characteristic map from [7].

\section{Eilenberg-Zilber isomorphisms}

In this section we recall the Eilenberg-Zilber isomorphisms for Hochschild, cyclic and periodic cyclic (co)homology of (co)cyclic modules where they will be used crucially in future sections [1,10,21]. Given a (co)cyclic module $(C, \delta, s, \tau)$ [3,24], where $\delta, s$ and $\tau$ are (co)faces, (co)degeneracies and (co)cyclic maps respectively, we denote its Hochschild differential in cohomology by $b^{n}=\sum_{i=0}^{n}(-1)^{i} \delta_{i}$, and Connes' differential by $B^{n}=\left(\sum_{i=0}^{n}(-1)^{n i} \tau_{n}^{i}\right) s_{n} \tau_{n}\left(1-\tau_{n}\right)$. Any (co)cyclic module defines a mixed complex $(C, b, B)$ in a natural way [3,18,24]. Suppose $\left(C^{n}, \delta_{i}^{n}, s_{i}^{n}, \tau^{n}\right)$ and $\left(C^{\prime n}, \delta_{i}^{\prime n}, s_{i}^{\prime n}, \tau^{\prime n}\right)$ are two cocyclic modules. The diagonal $C \times C^{\prime}$ is the cocyclic module $\left(\left(C \times C^{\prime}\right)^{n}, \delta_{i}^{n} \otimes \delta_{i}^{\prime n}, s_{i}^{n} \otimes s_{i}^{\prime n}, \tau_{n} \otimes \tau_{n}^{\prime}\right)$ where

$$
\left(C \times C^{\prime}\right)^{n}=C^{n} \otimes C^{\prime n} .
$$

The tensor product complex $C \otimes C^{\prime}$ given by

$$
\left(C \otimes C^{\prime}\right)^{n}=\bigoplus_{p+q=n} C^{p} \otimes C^{\prime q},
$$

is not a cocyclic module but it has a mixed complex structure given by 


$$
b_{n}=\bigoplus_{i+j=n}\left(b_{i} \otimes i d_{j}+(-1)^{j} i d_{i} \otimes b_{j}^{\prime}\right) \text {, }
$$

and similarly for $B$.

We have two natural maps between these complexes. The Alexander-Whitney map

$$
A W_{n}:\left(C \otimes C^{\prime}\right)^{n} \longrightarrow\left(C \times C^{\prime}\right)^{n},
$$

is given by

$$
A W_{n}=\bigoplus_{i+j=n} A W_{i, j}
$$

where

$$
A W_{i, j}: C^{i} \otimes C^{\prime j} \longrightarrow C^{n} \otimes C^{\prime n}
$$

are defined by

$$
A W_{i, j}=\delta_{n}^{n} \delta_{n-1}^{n-1} \cdots \delta_{i+1}^{i+1} \otimes \delta_{0}^{n} \delta_{0}^{n-1} \cdots \delta_{0}^{i+1} .
$$

The Shuffle map

$$
s h_{n}:\left(C \times C^{\prime}\right)^{n} \longrightarrow\left(C \otimes C^{\prime}\right)^{n},
$$

is given by

$$
s h_{n}=\bigoplus_{i+j=n} s h_{i, j},
$$

where

$$
s h_{i, j}:\left(C \times C^{\prime}\right)^{n} \longrightarrow C^{i} \otimes C^{\prime j},
$$

are defined by

$$
s h_{i, j}=\sum_{\sigma} \operatorname{sign}(\sigma) s_{\sigma(i+1)}^{i} \cdots s_{\sigma(n)}^{n-1} \otimes s_{\sigma(1)}^{j} \cdots s_{\sigma(i)}^{n-1},
$$

and $\sigma$ runs over all $(i, j)$-shuffles. By an $(i, j)$ shuffle we mean a permutation $\sigma$ on elements $\{1,2, \ldots, n\}$ such that

$$
\sigma(1)<\sigma(2)<\cdots<\sigma(i), \quad \sigma(i+1)<\sigma(i+2)<\cdots<\sigma(n) .
$$

One has $[b, s h]=0$ and $[b, A W]=0$ in the normalized setting [3,24]. It can be shown that 


$$
s h \circ A W=i d, \quad A W \circ s h=b h+h b+i d,
$$

for some chain homotopy map $h$ [24]. So they induce inverse isomorphisms on Hochschild cohomology, called the Eilenberg-Zilber isomorphism:

$$
H H^{n}\left(C \otimes C^{\prime}\right) \cong H H^{n}\left(C \times C^{\prime}\right) \quad \forall n \geq 0 .
$$

In the dual case of cyclic modules, the map

$$
A W_{n}:\left(C \times C^{\prime}\right)_{n} \longrightarrow\left(C \otimes C^{\prime}\right)_{n},
$$

is given by

$$
A W_{n}=\bigoplus_{i+j=n} A W_{i, j},
$$

where $A W_{i, j}: C_{n} \otimes C_{n}^{\prime} \longrightarrow C_{i} \otimes C_{j}^{\prime}$ are given by

$$
A W_{i, j}=\delta_{i+1}^{i+1} \delta_{i+2}^{i+2} \cdots \delta_{n}^{n} \otimes \delta_{0}^{i+1} \delta_{0}^{i+2} \cdots \delta_{0}^{n} .
$$

Also

$$
s h_{n}:\left(C \otimes C^{\prime}\right)_{n} \longrightarrow\left(C \times C^{\prime}\right)_{n},
$$

is given by

$$
s h_{n}=\bigoplus_{i+j=n} s h_{i, j},
$$

where $s h_{i, j}: C_{i} \otimes C_{j}^{\prime} \longrightarrow C_{n} \otimes C_{n}^{\prime}$ can be defined as:

$$
s h_{i, j}=\sum_{\sigma} \operatorname{sign}(\sigma) s_{\sigma(n)}^{n-1} \cdots s_{\sigma(i+1)}^{i} \otimes s_{\sigma(i)}^{n-1} \cdots s_{\sigma(1)}^{j} .
$$

Now we state the Eilenberg-Zilber isomorphism for cyclic cohomology. To this end, we define another map namely the cyclic shuffle map as follows [23,24]. First we define an $(i, j)$-cyclic shuffle. Let $i, j, n \in \mathbb{N}$ with $n=i+j$. Consider the permutation $\sigma$ on the $n$ elements $\{1, \ldots, n\}$ obtained by first performing a cyclic permutation $p$ times on $\{1, \ldots, i\}$ and a cyclic permutation $q$ times on $\{i+1, \ldots, i+j\}$ and there after applying an $(i, j)$-shuffle to the combined result. We call $\sigma$ an $(i, j)$-cyclic shuffle if 1 appears before $i+1$ in the resulting sequence. One can define another map namely $s h^{\prime}$ which is in fact a cyclic version of the shuffle map:

$$
s h_{n}^{\prime}:\left(C \times C^{\prime}\right)^{n} \longrightarrow\left(C \otimes C^{\prime}\right)^{n-2},
$$


where

$$
s h_{n}^{\prime}=\bigoplus_{i+j=n} s h_{i, j}^{\prime}
$$

and $s h_{i, j}^{\prime}: C^{n} \otimes C^{\prime n} \longrightarrow C^{i-1} \otimes C^{\prime j-1}$, are given by

$s h_{i, j}^{\prime}=\sum_{\sigma} \operatorname{sign}(\sigma) s_{i-p-1}^{i-1} \tau_{i}^{p+1} s_{\sigma(i+1)}^{i} \cdots s_{\sigma(n)}^{n-1} \otimes s_{j-q-1}^{j-1} \tau_{j}^{q+1} s_{\sigma(1)}^{j} \cdots s_{\sigma(i)}^{n-1}$.

Here $\sigma$ runs over all $(i, j)$-cyclic shuffles.

We denote the $(b, B)$-bicomplex of a (co)cyclic module $C$ by $\mathcal{B}(C)$ and its total complex by $T$ ot $\mathcal{B}(C)$. It is a mixed complex. Recall that an $S$-map $[18,24]$ between mixed complexes is a map of complexes $f: \operatorname{Tot} \mathcal{B}(C) \longrightarrow \operatorname{Tot} \mathcal{B}\left(C^{\prime}\right)$ which commutes with Connes' periodicity map $S$. In fact an $S$-map has a matrix representation:

$$
\left(\begin{array}{cccc}
f_{0} & f_{1} & f_{2} & \ldots \\
f_{-1} & f_{0} & f_{1} & \ldots \\
f_{-2} & f_{-1} & f_{0} & \ldots \\
\vdots & \vdots & \ddots &
\end{array}\right)
$$

where $f_{i}: C^{*} \longrightarrow C^{*-2 i}$, and

$$
\left[B, f_{i+1}\right]+\left[b, f_{i}\right]=0 .
$$

An $S$-map induces a map on the level of cyclic cohomology. Now we define the desired $S$-map $\widetilde{S h}: T o t \mathcal{B}\left(C \times C^{\prime}\right) \longrightarrow$ Tot $\mathcal{B}\left(C \otimes C^{\prime}\right)$ as:

$$
\widetilde{S h}=\left(\begin{array}{cccc}
s h & s h^{\prime} & 0 & \ldots \\
0 & s h & s h^{\prime} & \ldots \\
0 & 0 & s h & \ldots \\
\vdots & \vdots & \ddots &
\end{array}\right)
$$

The condition (2.13) reduces to the following relations in the normalized setting:

(1) $[b, s h]=0$,

(2) $[B, s h]+\left[b, s h^{\prime}\right]=0$,

(3) $\left[B, s h^{\prime}\right]=0$.

Also there is another $S$-map $\widetilde{A W}: \operatorname{Tot} \mathcal{B}\left(C \otimes C^{\prime}\right) \longrightarrow \operatorname{Tot} \mathcal{B}\left(C \times C^{\prime}\right)$, given by

$$
\widetilde{A W}=\left(\begin{array}{cccc}
A W & A W^{\prime} & 0 & \ldots \\
0 & A W & A W^{\prime} & \ldots \\
0 & 0 & A W & \ldots \\
\vdots & \vdots & \ddots &
\end{array}\right)
$$

Here $A W_{n}^{\prime}=\varphi_{n} B_{n} A W_{n}:\left(C \otimes C^{\prime}\right)^{n} \longrightarrow\left(C \times C^{\prime}\right)^{n-2}$, where $[1,26]$ 


$$
\begin{aligned}
\varphi_{n}= & \sum_{\otimes\left(s_{\alpha_{p+1}+n-p-q}\right.}(-1)^{n-p-q+\sigma(\alpha, \beta)}\left(s_{\beta_{q}+n-p-q} \cdots s_{\alpha_{1}+n-p-q} \delta_{n-p-q} \cdots \delta_{n-q-1}\right) .
\end{aligned}
$$

The sum is taken over all $0 \leq q \leq n-1,0 \leq p \leq n-q-1$, and $(\alpha, \beta)$ runs over all $(p+1, q)$-shuffles where $\sigma(\alpha, \beta)=\sum\left(\alpha_{i}-i+1\right)$. One can show [1]

$$
\widetilde{S h} \circ \widetilde{A W}=i d, \quad \widetilde{A W} \circ \widetilde{S h}=(b+B) \circ h+h \circ(b+B)+i d,
$$

for some homotopy map $h: C \otimes C^{\prime} \longrightarrow C \times C^{\prime}$. Thus we obtain the Eilenberg-Zilber isomorphism in cyclic cohomology $[1,21,24]$ :

$$
H C^{*}\left(C \times C^{\prime}\right) \cong H C^{*}\left(C \otimes C^{\prime}\right) .
$$

In the case of cyclic modules, one can define

$$
s h_{n}^{\prime}:\left(C \otimes C^{\prime}\right)_{n} \longrightarrow\left(C \times C^{\prime}\right)_{n+2} \quad \forall n \geq 0
$$

and

$$
A W_{n}^{\prime}:\left(C \times C^{\prime}\right)_{n} \longrightarrow\left(C \otimes C^{\prime}\right)_{n+2} \quad \forall n \geq 0 .
$$

This enables us to have the $S$-maps $\widetilde{S h}: \operatorname{Tot} \mathcal{B}\left(C \otimes C^{\prime}\right) \longrightarrow \operatorname{Tot} \mathcal{B}\left(C \times C^{\prime}\right)$, and $\widetilde{A W}: \operatorname{Tot} \mathcal{B}\left(C \times C^{\prime}\right) \longrightarrow \operatorname{Tot} \mathcal{B}\left(C \otimes C^{\prime}\right)$ which induce the Eilenberg-Zilber isomorphism in cyclic homology.

We have the Eilenberg-Zilber isomorphism for periodic cyclic cohomology as follows. One knows that $\lim _{\longrightarrow} \operatorname{Tot}^{2 n+*} \mathcal{B}(C)=\oplus_{n \geq 0} C^{2 n+*}$, where $*=0,1$ and the direct limit is with respect to Connes' periodicity map $S$. Since direct limit commutes with homology we have

$$
\begin{aligned}
\lim _{\longrightarrow} H C^{2 n+*}(C) & =\lim _{\longrightarrow} H\left(T o t^{2 n+*} \mathcal{B}(C)\right)=H\left(\underline{\lim }_{\longrightarrow} T t^{2 n+*} \mathcal{B}(C)\right) \\
& =H\left(\oplus_{n \geq 0} C^{2 n+*}\right)=H P^{*}(C),
\end{aligned}
$$

where $*=0,1$. Since

$H P^{*}\left(C \otimes C^{\prime}\right)=\lim _{\longrightarrow} H C^{2 n+*}\left(C \otimes C^{\prime}\right) \cong \lim _{\longrightarrow} H C^{2 n+*}\left(C \times C^{\prime}\right)=H P^{*}\left(C \times C^{\prime}\right)$,

we obtain the Eilenberg-Zilber isomorphism for periodic cyclic cohomology:

$$
H P^{*}\left(C \otimes C^{\prime}\right) \cong H P^{*}\left(C \times C^{\prime}\right),
$$

The explicit maps which induce the isomorphism (2.19), are infinite matrix versions of $\widetilde{S h}$ and $\widetilde{A W}$.

There is an obstacle to get the Eilenberg-Zilber isomorphism for periodic cyclic homology. The problem comes from the fact that homology does not commute with 
inverse limit in general. The Mittag-Leffler condition [9] on the inverse system $(H C(C)[-2 m], S)_{m}$ guarantees the commutativity of homology and inverse limit. If we have this condition, with a similar argument we obtain

$$
H P_{n}\left(C \otimes C^{\prime}\right) \cong H P_{n}\left(C \times C^{\prime}\right) .
$$

\section{Künneth formulas}

In this section we recall the Künneth formula for Hochschild and cyclic (co)homology of (co)cyclic modules and introduce a formula for the periodic case. If $C$ and $C^{\prime}$ are cocyclic objects in the category of vector spaces, we have the following Künneth formula:

$$
H H^{n}\left(C \otimes C^{\prime}\right) \cong \bigoplus_{p+q=n} H H^{p}(C) \otimes H H^{q}\left(C^{\prime}\right) \quad \forall n \geq 0 .
$$

The above isomorphism is induced by the shuffle map and following natural map [24]

$$
\mathfrak{I}:[\alpha] \otimes[\beta] \longmapsto[\alpha \otimes \beta] .
$$

The same maps induce an isomorphism for Hochschild homology of cyclic modules.

In the case of cyclic homology we do not have an isomorphism similar to (3.1). Instead, there is a short exact sequence $[2,16,19,24]$ :

$$
\begin{aligned}
0 \longrightarrow \operatorname{Tot}_{n} \mathcal{B}\left(C \otimes C^{\prime}\right) \stackrel{\mathcal{I}}{\longrightarrow} & \bigoplus{ }_{i+j=n} \operatorname{Tot}_{i} \mathcal{B}(C) \otimes \operatorname{Tot}_{j} \mathcal{B}\left(C^{\prime}\right) \stackrel{S \otimes i d-i d \otimes S}{\longrightarrow} \\
& \bigoplus{ }_{i+j=n-2} \operatorname{Tot}_{i} \mathcal{B}(C) \otimes \operatorname{Tot}_{j} \mathcal{B}\left(C^{\prime}\right) \longrightarrow 0 .
\end{aligned}
$$

The map $\mathcal{I}$ is called the Künneth map which can be defined as follows. Let $\operatorname{Tot} \mathcal{B}(C)=k[u] \otimes C$, where $|u|=2, \operatorname{Tot} \mathcal{B}\left(C^{\prime}\right)=k\left[u^{\prime}\right] \otimes C^{\prime}$ where $\left|u^{\prime}\right|=2$, and $\operatorname{Tot} \mathcal{B}\left(C \otimes C^{\prime}\right)=k[v] \otimes C \otimes C^{\prime}$ where $|v|=2$. One can define the map $\mathcal{I}$ as $[2,24]$ :

$$
\mathcal{I}\left(v^{n}\right)=\sum_{p+q=n} u^{p} u^{\prime q}
$$

From the Künneth short exact sequence (3.3), we get the following long exact sequence in homology:

$$
\begin{aligned}
\ldots \longrightarrow H C_{n}\left(C \otimes C^{\prime}\right) \stackrel{\mathcal{I}}{\longrightarrow} \bigoplus_{i+j=n} H C_{i}(C) \otimes H C_{j}\left(C^{\prime}\right) \stackrel{S \otimes i d-i d \otimes S}{\longrightarrow} \\
\bigoplus_{i+j=n-2} H C_{i}(C) \otimes H C_{j}\left(C^{\prime}\right) \stackrel{\partial}{\longrightarrow} H C_{n-1}\left(C \otimes C^{\prime}\right) \longrightarrow \ldots
\end{aligned}
$$


Similarly for cyclic cohomology, we have the following short exact sequence:

$$
0 \longrightarrow T o t^{n} B\left(C \otimes C^{\prime}\right) \stackrel{\mathcal{I}}{\longrightarrow} \bigoplus_{i+j=n} T t^{i} B(C) \otimes T o t^{j} B\left(C^{\prime}\right) \stackrel{S \otimes i d-i d \otimes S}{\longrightarrow}
$$

$$
\bigoplus_{i+j=n+2} \operatorname{Tot}^{i} B(C) \otimes \operatorname{Tot}^{j} B\left(C^{\prime}\right) \longrightarrow 0
$$

Consequently, we get the following long exact sequence in cohomology $[2,18]$ :

$$
\begin{gathered}
\ldots \longrightarrow H C^{n}\left(C \otimes C^{\prime}\right) \stackrel{\mathcal{I}}{\longrightarrow} \bigoplus_{p+q=n} H C^{p}(C) \otimes H C^{q}\left(C^{\prime}\right) \stackrel{S \otimes i d-i d \otimes S}{\longrightarrow} \\
\bigoplus_{r+s=n+2} H C^{r}(C) \otimes H C^{s}\left(C^{\prime}\right) \stackrel{\partial}{\longrightarrow} H C^{n+2}\left(C \otimes C^{\prime}\right) \longrightarrow \ldots
\end{gathered}
$$

where $\partial$ is the connecting morphism of short exact sequences.

Now we state the Künneth formula for the periodic case. One can find the formula for algebras in [8]. Here we provide a similar argument in the general case of (co)cyclic modules. Recall that a supercomplex is a $\mathbb{Z}_{2}$-graded vector space $V=V_{0} \oplus V_{1}$ endowed with linear maps $\partial_{0}: V_{0} \longrightarrow V_{1}$ and $\partial_{1}: V_{1} \longrightarrow V_{0}$ such that $\partial_{0} \partial_{1}=0$ and $\partial_{1} \partial_{0}=0$. We denote the corresponding chain complex by $V_{0} \underset{\partial_{1}}{\stackrel{\partial_{0}}{\leftrightarrows}} V_{1}$, where its homology is a $\mathbb{Z}_{2}$-graded vector space given by:

$$
H_{0}=\operatorname{Ker} \partial_{0} / \operatorname{Im} \partial_{1}, \quad H_{1}=\operatorname{Ker} \partial_{1} / \operatorname{Im} \partial_{0} .
$$

For example the inverse limit $\lim _{\leftarrow} \operatorname{Tot} \mathcal{B}(C)$ is a supercomplex. In this case $V=$ Tot $B_{n}(C), \partial=B+b$ and we have $\lim _{\longleftarrow} \operatorname{Tot} \mathcal{B}(C)=\left(\prod C_{2 n}\right)_{n \geq 0} \oplus\left(\prod C_{2 n+1}\right)_{n \geq 0}$. For cohomology, $\lim _{\longrightarrow} \operatorname{Tot} \mathcal{B}(C)=V_{0} \oplus V_{1}=\left(\oplus C^{2 n}\right)_{n \geq 0} \oplus\left(\oplus C^{2 n+1}\right)_{n \geq 0}$. A map of supercomplexes is a linear map $f: V=V_{0} \oplus V_{1} \longrightarrow W=W_{0} \oplus W_{1}$ such that sends $V_{0}$ to $W_{0}$ and $V_{1}$ to $W_{1}$. If we have two supercomplexes $V$ and $W$, then $V \widehat{\otimes} W$ is a supercomplex where $(V \widehat{\otimes} W)_{0}=\left(V_{0} \otimes W_{0}\right) \oplus\left(V_{1} \otimes W_{1}\right)$ and $(V \widehat{\otimes} W)_{1}=$ $\left(V_{0} \otimes W_{1}\right) \oplus\left(V_{1} \otimes W_{0}\right)$. We define

$$
\partial_{0}^{V \widehat{\otimes W}}=\left(\begin{array}{cc}
1 \otimes \partial_{0}^{W} & \partial_{1}^{V} \otimes 1 \\
\partial_{0}^{V} \otimes 1 & -1 \otimes \partial_{1}^{W}
\end{array}\right) \quad \text { and } \quad \partial_{1}^{V \widehat{\otimes W}}=\left(\begin{array}{cc}
1 \otimes \partial_{1}^{W} & \partial_{1}^{V} \otimes 1 \\
\partial_{0}^{V} \otimes 1 & -1 \otimes \partial_{0}^{W}
\end{array}\right)
$$

The Künneth formula for supercomplexes holds:

$$
H(X \widehat{\otimes} Y) \cong H(X) \widehat{\otimes} H(Y) .
$$

We define the supercomplex map:

$$
\nabla:\left(\lim _{\longleftarrow}^{\operatorname{lig}} \operatorname{\mathcal {B}}(\bar{C})\right) \widehat{\otimes}\left(\lim _{\longleftarrow}^{\lim } \operatorname{ot} \mathcal{B}\left(\overline{C^{\prime}}\right)\right) \longrightarrow \lim _{\longleftarrow} \operatorname{ot} \mathcal{B}\left(\bar{C} \otimes \overline{C^{\prime}}\right) .
$$


The restriction of $\nabla$ on $\left(\prod_{n \geq 0} C^{2 n}\right) \otimes\left(\prod_{n \geq 0} C^{2 n}\right)$ sends $\left\{\xi_{n}\right\}_{n} \otimes\left\{\xi_{n}^{\prime}\right\}_{n}$ onto $\left\{\sum_{i+j=n} \xi_{i} \otimes \xi_{j}\right\}_{n}$. Similarly one can define the restriction of $I$ on $\left(\prod_{n \geq 0} C^{2 n+1}\right) \otimes$ $\left(\prod_{n \geq 0} C^{\prime 2 n+1}\right),\left(\prod_{n \geq 0} C^{2 n}\right) \otimes\left(\prod_{n \geq 0} C^{\prime 2 n+1}\right)$ and $\left(\prod_{n \geq 0} C^{2 n+1}\right) \otimes\left(\prod_{n \geq 0} C^{\prime 2 n}\right)$. To prove that $\nabla$ is a quasi-isomorphism, we have two major problems. The first one is the fact that generally homology does not commute with inverse limit, i.e., $H_{*}\left(\lim Z_{m}\right) \neq \lim H_{*}\left(Z_{m}\right)$, where $Z_{m}$ is an inverse system. To solve this problem we need the Mittag-Leffler condition which guarantees the commutativity. The second problem is in general $\left(\prod_{i=1}^{\infty} V_{i}\right) \otimes W \nsucceq \prod_{i=1}^{\infty}\left(V_{i} \otimes W\right)$, where $V_{i}$ and $W$ are some vector spaces. If $W$ is finite dimensional then we obtain an isomorphism. In our case we can think about $V_{i}$ and $W$ as the homology of a cyclic module. Now we are ready to have the following theorem:

Theorem 3.1 Suppose $C$ is a cyclic module which has the following two properties:

(i) The inverse system $(H C(C)[-2 m], S)_{m}$ satisfies the Mittag-Leffler condition.

(ii) The periodic cyclic homology $H P_{*}(C)$ is a finite dimensional vector space.

Then, the map

$$
\nabla: H P_{*}(C) \widehat{\otimes} H P_{*}\left(C^{\prime}\right) \longrightarrow H P_{*}\left(C \otimes C^{\prime}\right)
$$

is an isomorphism for any cyclic module $C^{\prime}$.

Proof The proof is similar to the one in [8] for algebras. Here we just mention that the condition (i) gives us $H P(C)=\underset{\longleftarrow}{\lim } H C(C)[-2 m]$ and (ii) proves

$$
\left(H P(C) \widehat{\otimes} \prod H C\left(C^{\prime}\right)[-2 m]\right)_{*} \cong \prod\left(H P_{*-[m]}(C) \otimes H C_{m}\left(C^{\prime}\right)\right) .
$$

Thus under the assumptions of the Theorem 3.1, one obtains the Künneth formula for periodic cyclic homology as follows:

$$
H P_{0}(C) \otimes H P_{0}\left(C^{\prime}\right) \oplus H P_{1}(C) \otimes H P_{1}\left(C^{\prime}\right) \cong H P_{0}\left(C \otimes C^{\prime}\right),
$$

and

$$
H P_{0}(C) \otimes H P_{1}\left(C^{\prime}\right) \oplus H P_{1}(C) \otimes H P_{0}\left(C^{\prime}\right) \cong H P_{1}\left(C \otimes C^{\prime}\right)
$$

For periodic cyclic cohomology, since direct limit commutes with cohomology, we do not need (i) and also since $\left.\lim _{\longrightarrow} \operatorname{Tot} \mathcal{B}(C)=\left(\oplus C^{2 n}\right)_{n \geq 0} \oplus\left(\oplus C^{2 n+1}\right)_{n \geq 0}\right)$ and $\oplus_{i \geq 0}\left(V_{i} \otimes W\right) \simeq\left(\oplus_{i \geq 0} V_{i}\right) \otimes W$, for all vector spaces $V_{i}$ and $W$, we do not need (ii). Therefore we have the following theorem:

Theorem 3.2 Suppose $C$ and $C^{\prime}$ are two cocyclic modules. Then, the map

$$
\nabla: H P^{*}(C) \widehat{\otimes} H P^{*}\left(C^{\prime}\right) \longrightarrow H P^{*}\left(C \otimes C^{\prime}\right),
$$

is an isomorphism and we have the relations (3.9) and (3.10) in cohomology. 


\section{Eilenberg-Zilber isomorphisms and Künneth formulas in Hopf cyclic theory}

In this section we establish the Eilenberg-Zilber isomorphisms and Künneth formulas for Hopf cyclic theory with coefficients. Throughout the paper we assume that $\mathcal{H}$ is a Hopf algebra with a bijective antipode over a field $k$ of characteristic zero. The coproduct, counit and antipode of $\mathcal{H}$ are denoted by $\Delta, \varepsilon$ and $S$, respectively. For the coproduct we use the Sweedler notation in the form $\Delta(h)=h^{(1)} \otimes h^{(2)}$; for a left coaction of $\mathcal{H}$ on a comodule $M, \boldsymbol{\nabla}: M \longrightarrow \mathcal{H} \otimes M$, we write $\boldsymbol{\nabla}(m)=m^{(-1)} \otimes m^{(0)}$, and for a right coaction we write $\boldsymbol{\nabla}(m)=m^{(0)} \otimes m^{(1)}$. If $f$ is a map of (co)chain complexes, then $f^{*}$ denotes the induced map on (co)homology.

Definition 4.1 [12] Let $\mathcal{H}$ be a Hopf algebra and $M$ a right module and a left comodule over $\mathcal{H}$. We call $M$ an anti-Yetter-Drinfeld module, if the action and coaction are compatible in the following sense:

$$
(m h)^{(-1)} \otimes(m h)^{(0)}=S\left(h^{(3)}\right) m^{(-1)} h^{(1)} \otimes m^{(0)} h^{(2)},
$$

for all $m \in M$ and $h \in \mathcal{H}$. We call $M$ stable if for all $m \in M, m^{(-1)} m^{(0)}=m$.

We abbreviate stable anti-Yetter-Drinfeld by SAYD. For example, given a character, i.e., a unital algebra map $\delta: \mathcal{H} \longrightarrow k$, and a group-like element $\sigma \in \mathcal{H}$, one defines a SAYD module $M={ }^{\sigma} k_{\delta}$, where the action of $\mathcal{H}$ is defined by the character $\delta$, $m h=\delta(h) m$, and the coaction via the group-like element $\sigma, \boldsymbol{\nabla}(m)=\sigma \otimes m$. Then $M={ }^{\sigma} k_{\delta}$ is stable if and only if $(\delta, \sigma)$ is a modular pair, i.e., $\delta(\sigma)=1$, and anti-YetterDrinfeld if and only if $(\delta, \sigma)$ is in involution, i.e., $\sigma^{-1} \widetilde{S}^{2}(h) \sigma=h, \quad \forall h \in \mathcal{H}$, where $\widetilde{S}(h):=\delta\left(h^{(1)}\right) S\left(h^{(2)}\right)$ [7]. This module is called a modular pair in involution.

Given a Hopf algebra $\mathcal{H}$ equipped with a SAYD $\mathcal{H}$-module $M$, we have the cocyclic module $C(\mathcal{H}, M)$ where $C^{n}(\mathcal{H}, M)=M \otimes_{\mathcal{H}} \mathcal{H}^{\otimes(n+1)}, n \geq 0$. Its cofaces, codegeneracies and cocyclic map are as follows [13]:

$$
\begin{aligned}
\delta_{i}\left(m \otimes \mathcal{H} h_{0} \otimes \cdots \otimes h_{n-1}\right) & =m \otimes \mathcal{H} h_{0} \otimes \cdots \otimes h_{i}^{(1)} \otimes h_{i}^{(2)} \cdots \otimes h_{n-1}, \quad 0 \leq i<n \quad \text { (cofaces), } \\
\delta_{n}\left(m \otimes \mathcal{H} h_{0} \otimes \cdots \otimes h_{n-1}\right) & =m^{(0)} \otimes \mathcal{H} h_{0}^{(2)} \otimes h_{1} \otimes \cdots \otimes h_{n-1} \otimes m^{(-1)} h_{0}^{(1)} \quad \text { (flip-over face), } \\
\sigma_{i}\left(m \otimes \mathcal{H} h_{0} \otimes \cdots \otimes h_{n+1}\right) & =m \otimes \mathcal{H} h_{0} \otimes \cdots \otimes \varepsilon\left(h_{i+1}\right) \otimes \cdots \otimes h_{n+1}, \quad 0 \leq i \leq n \text { (codegeneracies), } \\
\tau_{n}\left(m \otimes \mathcal{H} h_{0} \otimes \cdots \otimes h_{n}\right) & =m^{(0)} \otimes \mathcal{H} h_{1} \otimes \cdots \otimes h_{n} \otimes m^{(-1)} h_{0} \quad \text { (cocyclic map). }
\end{aligned}
$$

For $M={ }^{\sigma} k_{\delta}$, the complex $C(\mathcal{H}, M)$ reduces to the cocyclic module of ConnesMoscovici [7].

Lemma 4.1 If $\mathcal{H}$ and $\mathcal{K}$ are Hopf algebras and $M$ and $N$, SAYD modules over $\mathcal{H}$ and $\mathcal{K}$ respectively, then $M \otimes N$ is a $S A Y D$ module over $\mathcal{H} \otimes \mathcal{K}$ in a natural way.

Proof We define the right action by:

$$
(m \otimes n)(h \otimes k)=m h \otimes n k,
$$

and the left coaction by:

$$
\boldsymbol{\nabla}(m \otimes n)=m^{(-1)} \otimes n^{(-1)} \otimes m^{(0)} \otimes n^{(0)} .
$$


We check the compatibility of action and coaction:

$$
\begin{aligned}
& \boldsymbol{\nabla}((m \otimes n)(h \otimes k))=\mathbf{\nabla}(m h \otimes n k)=(m h \otimes n k)^{(-1)} \otimes(m h \otimes n k)^{(0)} \\
& \quad=(m h)^{(-1)} \otimes(n k)^{(-1)} \otimes(m h)^{(0)} \otimes(n k)^{(0)} \\
& \quad=S\left(h^{(3)}\right) m^{(-1)} h^{(1)} \otimes S\left(k^{(3)}\right) n^{(-1)} k^{(1)} \otimes m^{(0)} h^{(2)} \otimes n^{(0)} k^{(2)} \\
& \quad=S_{\mathcal{H} \otimes \mathcal{K}}\left(h^{(3)} \otimes k^{(3)}\right)(m \otimes n)^{(-1)}\left(h^{(1)} \otimes\left(k^{(1)}\right) \otimes\left((m \otimes n)^{(0)}\right)\left(h^{(2)} \otimes k^{(2)}\right) .\right.
\end{aligned}
$$

To check the stability:

$$
\left(m^{(0)} \otimes n^{(0)}\right)\left(m^{(-1)} \otimes n^{(-1)}\right)=\left(m^{(0)} m^{(-1)} \otimes n^{(0)} n^{(-1)}\right)=(m \otimes n) .
$$

We need the following statement later.

Lemma 4.2 If $\mathcal{H}$ and $\mathcal{K}$ are Hopf algebras and $M$ and $N$ SAYD modules over $\mathcal{H}$ and $\mathcal{K}$ respectively, then the following map

$\Omega_{r}:(M \otimes N) \otimes \mathcal{H} \otimes \mathcal{K}(\mathcal{H} \otimes \mathcal{K})^{\otimes r+1} \longrightarrow\left(M \otimes_{\mathcal{H}} \mathcal{H}^{\otimes r+1}\right) \otimes\left(N \otimes \mathcal{K} \mathcal{K}^{\otimes r+1}\right), r \geq 0$

given by

$$
\begin{aligned}
& \left((m \otimes n) \otimes \mathcal{H} \otimes \mathcal{K}\left(h_{0} \otimes k_{0}\right) \otimes \cdots \otimes\left(h_{r} \otimes k_{r}\right)\right) \\
& \quad \longmapsto\left(m \otimes \mathcal{H}_{0} \otimes \cdots \otimes h_{r}\right) \otimes\left(n \otimes \mathcal{K} k_{0} \otimes \cdots \otimes k_{r}\right),
\end{aligned}
$$

is well-defined and defines an isomorphism of cocyclic modules:

$$
C(\mathcal{H} \otimes \mathcal{K}, M \otimes N) \cong C(\mathcal{H}, M) \times C(\mathcal{K}, N)
$$

Proof Since we consider $\mathcal{H}, \mathcal{K}$ and $\mathcal{H} \otimes \mathcal{K}$ as $\mathcal{H}^{\otimes m}, \mathcal{K}^{\otimes m}$ and $(\mathcal{H} \otimes \mathcal{K})^{\otimes m}$-modules, respectively, by diagonal action, the map $\Omega$ is well-defined. We show that $\Omega_{r}$ commutes with $\delta_{i}$ and $\tau_{r}$, where $0 \leq i<r$. The commutativity of $\Omega_{r}$ with $\delta_{r}$ and $\sigma_{i}$ 's is left to the reader. For $\delta_{i}$ 's we have:

$$
\begin{aligned}
\Omega_{r} & \delta_{i}((m \otimes n) \otimes \mathcal{H} \otimes \mathcal{K} \\
= & \Omega_{r}\left((m \otimes n) \otimes \mathcal{H} \otimes \mathcal{K}\left(h_{0} \otimes k_{0}\right) \otimes \cdots \otimes\left(h_{r-1} \otimes k_{r-1}\right)\right) \\
& \otimes \cdots \otimes\left(h_{r-1} \otimes k_{r-1}\right) \\
= & \Omega_{r}\left((m \otimes n) \otimes \mathcal{H} \otimes \mathcal{K}\left(h_{0} \otimes k_{0}\right) \otimes \ldots \otimes\left(h_{i}^{(1)} \otimes k_{i}\right)^{(1)} \otimes\left(h_{i} \otimes k_{i}\right)^{(2)} \otimes\left(h_{i}^{(2)} \otimes k_{i}^{(2)}\right)\right. \\
& \left.\otimes \ldots\left(h_{r-1} \otimes k_{r-1}\right)\right) \\
= & \left(m \otimes \mathcal{H} h_{0} \otimes \cdots \otimes h_{i}^{(1)} \otimes h_{i}^{(2)} \otimes \ldots \otimes h_{r-1}\right) \otimes\left(n \otimes \mathcal{K} k_{0} \otimes \cdots \otimes k_{i}^{(1)} \otimes k_{i}^{(2)}\right. \\
& \left.\otimes \ldots \otimes k_{r-1}\right) \\
= & \left(\delta_{i} \otimes \delta_{i}\right)\left(m \otimes \mathcal{H} h_{0} \otimes \cdots \otimes h_{r-1}\right) \otimes\left(n \otimes \mathcal{K} k_{0} \otimes \cdots \otimes k_{r-1}\right) \\
= & \left(\delta_{i} \otimes \delta_{i}\right) \Omega_{r}\left((m \otimes n) \otimes \mathcal{H} \otimes \mathcal{K}\left(h_{0} \otimes k_{0}\right) \otimes \cdots \otimes\left(h_{r-1} \otimes k_{r-1}\right)\right) .
\end{aligned}
$$


For $\tau_{r}$ :

$$
\begin{aligned}
\Omega_{r} & \tau_{r}\left((m \otimes n) \otimes \mathcal{H} \otimes \mathcal{K}\left(h_{0} \otimes k_{0}\right) \otimes \cdots \otimes\left(h_{r} \otimes k_{r}\right)\right) \\
= & \Omega_{r}\left(\left(m^{(0)} \otimes n^{(0)}\right) \otimes \mathcal{H} \otimes \mathcal{K}\left(h_{0} \otimes k_{0}\right)\right. \\
& \otimes \cdots \otimes\left(h_{r} \otimes k_{r}\right) \otimes\left(\left(m^{(-1)} \otimes n^{(-1)}\right)\left(h_{0} \otimes k_{0}\right)\right) \\
& =\left(m^{(0)} \otimes \mathcal{H} h_{1} \otimes \cdots \otimes h_{r} \otimes m^{(-1)} h_{0}\right) \otimes\left(n^{(0)} \otimes \mathcal{K} k_{1} \otimes \cdots \otimes k_{r} \otimes n^{(-1)} k_{0}\right) \\
= & \left(\tau_{r} \otimes \tau_{r}\right)\left(\left(m \otimes \mathcal{H} h_{0} \otimes \cdots \otimes h_{r}\right) \otimes\left(n \otimes \mathcal{K} k_{0} \otimes \cdots \otimes k_{r}\right)\right. \\
= & \left(\tau_{r} \otimes \tau_{r}\right) \Omega_{r}\left(\left((m \otimes n) \otimes \mathcal{H} \otimes \mathcal{K}\left(h_{0} \otimes k_{0}\right) \otimes \cdots \otimes\left(h_{r} \otimes k_{r}\right)\right) .\right.
\end{aligned}
$$

The bijectivity of $\Omega_{r}$ is obvious.

Using the Eilenberg-Zilber isomorphisms (2.5), (2.16), (2.19), Künneth formulas (3.1), (3.11), coupled with the above two lemmas, we obtain the following propositions.

Proposition 4.1 Let $\mathcal{H}$ and $\mathcal{K}$ be two Hopf algebras and $M$ and $N$ be SAYD modules over $\mathcal{H}$ and $\mathcal{K}$ respectively. We have the the following isomorphisms for Hopf Hochschild, cyclic, and periodic cyclic cohomology of Hopf algebras with coefficients:

$$
\begin{aligned}
H H^{*}(\mathcal{H} \otimes \mathcal{K}, M \otimes N) & \cong H H^{*}(C(\mathcal{H}, M) \otimes C(\mathcal{K}, N)), \\
H C^{*}(\mathcal{H} \otimes \mathcal{K}, M \otimes N) & \cong H C^{*}(C(\mathcal{H}, M) \otimes C(\mathcal{K}, N)), \\
H P^{*}(\mathcal{H} \otimes \mathcal{K}, M \otimes N) & \cong H P^{*}(C(\mathcal{H}, M) \otimes C(\mathcal{K}, N)) .
\end{aligned}
$$

Proposition 4.2 Let $\mathcal{H}$ and $\mathcal{K}$ be two Hopf algebras and $M$ and $N$ be SAYD modules over $\mathcal{H}$ and $\mathcal{K}$ respectively. We have the following isomorphisms of vector spaces,

$$
H H^{n}(\mathcal{H} \otimes \mathcal{K}, M \otimes N) \cong \bigoplus_{i+j=n} H H^{i}(\mathcal{H}, M) \otimes H H^{j}(\mathcal{K}, N)
$$

and

$$
H P^{n}(\mathcal{H} \otimes \mathcal{K}, M \otimes N) \cong \bigoplus_{i+j=n} H P^{i}(\mathcal{H}, M) \otimes H P^{j}(\mathcal{K}, N), \quad n=0,1
$$

and the following long exact sequence of differential graded vector spaces,

$$
\begin{aligned}
\ldots \longrightarrow H C^{n}(\mathcal{H} \otimes \mathcal{K}, M \otimes N) & \stackrel{\mathcal{I}}{\longrightarrow} \bigoplus_{p+q=n} H C^{p}(\mathcal{H}, M) \otimes H C^{q}(\mathcal{K}, N) \stackrel{S \otimes i d-i d \otimes S}{\longrightarrow} \\
\bigoplus_{r+s=n+2} H C^{r}(\mathcal{H}, M) \otimes H C^{s}(\mathcal{K}, N) & \stackrel{\partial}{\longrightarrow} H C^{n+2}(\mathcal{H} \otimes \mathcal{K}, M \otimes N) \longrightarrow \ldots
\end{aligned}
$$

\section{Coproducts in Hopf cyclic cohomology}

In this section we define coproducts for Hopf Hochschild, cyclic and periodic cyclic cohomology of cocommutative Hopf algebras. The following statement plays an important role in the definition of these coproducts. 
Proposition 5.1 Let $\mathcal{H}$ be a cocommutative Hopf algebra and M a SAYD module over $\mathcal{H}$. Let

$$
\Phi_{n}=\psi \otimes \Delta^{\otimes n+1}: C^{n}(\mathcal{H}, M) \longrightarrow C^{n}(\mathcal{H} \otimes \mathcal{H}, M \otimes M)
$$

be a linear map, where $\psi: M \longrightarrow M \otimes M$, satisfying the following condition

$$
(\Delta \otimes \psi) \circ \boldsymbol{\nabla}_{M}=\boldsymbol{\nabla}_{M \otimes M} \circ \psi
$$

Then the map

$$
\rho_{n}=\Omega_{n} \Phi_{n}: C^{n}(\mathcal{H}, M) \longrightarrow(C(\mathcal{H}, M) \times C(\mathcal{H}, M))^{n}
$$

is a map of cocyclic modules.

Proof Since $\mathcal{H}$ is cocommutative, one can easily see that $\rho_{n} \delta_{i}=\left(\delta_{i} \otimes \delta_{i}\right) \rho_{n}$ where $0 \leq i \leq n-1$. We show that $\rho_{n}$ commutes with $\delta_{n}$. For this we use the summation notation $\psi(m)=m_{(1)} \otimes m_{(2)}$. The condition (5.1) is equivalent to:

$$
\begin{aligned}
& \left(m_{(1)}\right)^{(-1)} \otimes\left(m_{(2)}\right)^{(-1)} \otimes\left(m_{(1)}\right)^{(0)} \otimes\left(m_{(2)}\right)^{(0)} \\
& \quad=m^{(-1)(1)} \otimes m^{(-1)(2)} \otimes\left(m^{(0)}\right)_{(1)} \otimes\left(m^{(0)}\right)_{(2)} .
\end{aligned}
$$

By cocommutativity of $\mathcal{H}$ we have:

$$
\begin{aligned}
&\left(\delta_{n} \otimes \delta_{n}\right) \Omega_{n-1}\left(\psi \otimes \Delta^{\otimes n}\right)\left(m \otimes h_{0} \otimes \cdots \otimes h_{n-1}\right) \\
&=\left(\delta_{n} \otimes \delta_{n}\right) \Omega_{n-1}\left(\psi(m) \otimes \Delta\left(h_{0}\right) \otimes \cdots \otimes \Delta\left(h_{n-1}\right)\right) \\
&=\left(\delta_{n} \otimes \delta_{n}\right)\left(m_{(1)} \otimes h_{1}^{(1)} \otimes \ldots h_{n-1}^{(1)}\right) \otimes\left(m_{(2)} \otimes h_{1}^{(2)} \otimes \ldots h_{n-1}^{(2)}\right) \\
&= \Omega_{n-1}\left(\left(m_{(1)}\right)^{(0)} \otimes h_{0}^{(1)(2)} \otimes h_{2}^{(1)} \otimes \cdots \otimes h_{n-1}^{(1)} \otimes\left(m_{(1)}\right)^{(-1)} h_{0}^{(1)(1)}\right) \otimes\left(\left(m_{(2)}\right)^{(0)}\right. \\
&\left.\otimes h_{0}^{(2)(2)} \otimes h_{1}^{(2)} \otimes \cdots \otimes h_{n-1}^{(2)} \otimes\left(m_{(2)}\right)^{(-1)} h_{0}^{(2)(1)}\right) \\
&= \Omega_{n-1}\left(\left(\left(m_{(1)}\right)^{(0)} \otimes\left(m_{(2)}\right)^{(0)}\right) \otimes\left(h_{0}^{(1)(2)} \otimes h_{0}^{(2)(2)}\right) \otimes\left(h_{1}^{(1)} \otimes h_{1}^{(2)}\right)\right. \\
&\left.\left.\otimes \cdots \otimes\left(h_{n-1}^{(1)} \otimes h_{n-1}^{(2)}\right) \otimes\left(m_{(1)}\right)^{(-1)} h_{0}^{(1)(1)} \otimes\left(m_{(2)}\right)^{(-1)} h_{0}^{(2)(1)}\right)\right) \\
&= \Omega_{n-1}\left(\psi(m)^{(0)} \otimes\left(\Delta\left(h_{1}\right)\right)^{(2)} \otimes \Delta\left(h_{2}\right) \otimes \cdots \otimes \Delta\left(h_{n-1}\right) \otimes \psi(m)^{(-1)}\left(\Delta\left(h_{1}\right)\right)^{(1)}\right) \\
&= \Omega_{n-1}\left(\psi\left(m^{(0)}\right) \otimes \Delta\left(h_{0}^{(2)}\right) \otimes \Delta\left(h_{1}\right) \otimes \cdots \otimes \Delta\left(h_{n-1}\right) \otimes \Delta\left(m^{(-1)}\right) \Delta\left(h_{0}^{(1)}\right)\right) \\
&= \Omega_{n-1}\left(\psi \otimes \Delta^{\otimes n}\right)\left(m^{(0)} \otimes h_{0}^{(2)} \otimes h_{1} \otimes \cdots \otimes h_{n-1} \otimes m^{(-1)} h_{0}^{(1)}\right) \\
&= \Omega_{n-1}\left(\psi \otimes \Delta^{\otimes n}\right) \delta_{n}\left(m \otimes h_{0} \otimes \cdots \otimes h_{n-1}\right) .
\end{aligned}
$$


We show that $\rho_{n}$ commutes with $\tau_{n}$ :

$$
\begin{aligned}
& \Omega_{n}\left(\psi \otimes \Delta^{\otimes n+1}\right) \tau_{n}\left(m \otimes h_{0} \otimes h_{1} \cdots \otimes h_{n}\right) \\
& \quad=\Omega_{n}\left(\psi \otimes \Delta^{\otimes n+1}\right)\left(m^{(0)} \otimes h_{1} \otimes \cdots \otimes h_{n} \otimes m^{(-1)} h_{0}\right) \\
& =\Omega_{n}\left(\psi\left(m^{0}\right)\right) \otimes \Delta\left(h_{1}\right) \otimes \cdots \otimes \Delta\left(h_{n}\right) \otimes \Delta\left(m^{(-1)} \Delta\left(h_{0}\right)\right) \\
& =\Omega_{n}\left(( ( m ^ { ( 0 ) } ) _ { ( 1 ) } \otimes ( m ^ { ( 0 ) } ) _ { ( 2 ) } ) \otimes ( h _ { 1 } ^ { ( 1 ) } \otimes h _ { 1 } ^ { ( 2 ) } ) \otimes \ldots \otimes ( h _ { n } ^ { ( 1 ) } \otimes h _ { n } ^ { ( 2 ) } ) \otimes \left(m^{(-1)(1)} h_{0}^{(1)}\right.\right. \\
& \left.\left.=\otimes m^{(-1)(2)} h_{0}^{(2)}\right)\right)\left(\left(m^{(0)}\right)_{(1)} \otimes h_{1}^{(1)} \otimes \cdots \otimes h_{n}^{(1)} \otimes m^{(-1)(1)} h_{0}^{(1)}\right) \otimes\left(\left(m^{(0)}\right)_{(2)}\right. \\
& \left.=\otimes h_{1}^{(2)} \otimes \cdots \otimes h_{n}^{(2)} \otimes m^{(-1)(2)} h_{0}^{(2)}\right)\left(\left(m_{(1)}\right)^{(0)} \otimes h_{1}^{(1)} \otimes \cdots \otimes h_{n}^{(1)}\right. \\
& \left.=\otimes m_{(1)}^{(-1)} h_{0}^{(1)}\right) \otimes\left(\left(m_{(2)}\right)^{(0)} \otimes h_{1}^{(2)} \otimes \cdots \otimes h_{n}^{(2)} \otimes m_{(2)}^{(-1)} h_{0}^{(2)}\right) \tau_{n}\left(m_{(1)} \otimes h_{0}^{(1)}\right. \\
& \left.=\otimes \cdots \otimes h_{n}^{(1)}\right) \otimes \tau_{n}\left(m_{(2)} \otimes h_{0}^{(2)} \otimes \cdots \otimes h_{n}^{(2)}\right)\left(\tau_{n} \otimes \tau_{n}\right) \Omega_{n}\left(\psi(m) \otimes \Delta\left(h_{0}\right)\right. \\
& \left.\left.=\otimes \cdots \otimes \Delta\left(h_{n}\right)\right)\left(\tau_{n} \otimes \tau_{n}\right) \Omega_{n}\left(\psi \otimes \Delta^{\otimes(n+1)}\right)\left(m \otimes \mathcal{H} h_{0} \otimes \cdots \otimes h_{n}\right)\right)
\end{aligned}
$$

So we have:

$$
\Omega_{n}\left(\psi \otimes \Delta^{\otimes(n+1)}\right) \tau_{n}=\left(\tau_{n} \otimes \tau_{n}\right) \Omega_{n}\left(\psi \otimes \Delta^{\otimes(n+1)}\right) .
$$

Based on the proof of the previous lemma, in fact the map $\Phi_{n}$ is a map of cocyclic modules. Now we have all the needed tools to define the desired coproducts. The following theorem provides a coproduct for Hochschild cohomology of a cocommutative Hopf algebra with coefficients in a SAYD module.

Proposition 5.2 Suppose $\mathcal{H}$ is a cocommutative Hopf algebra, $M$ a SAYD module over $\mathcal{H}$, and $\psi: M \longrightarrow M \otimes M$, a linear map satisfying (5.1). The following map

$$
\sqcup=(S h \rho)^{*}: H H^{n}(\mathcal{H}, M) \longrightarrow \bigoplus_{i+j=n} H H^{i}(\mathcal{H}, M) \otimes H H^{j}(\mathcal{H}, M),
$$

defines a coproduct for Hopf Hochschild cohomology where $\rho$ is defined in Proposition 5.1 .

Proof Since $H$ is cocommutative, by Proposition 5.1 the map

$$
\rho: C^{n}(H, M) \longrightarrow C^{n}(H, M) \otimes C^{n}(H, M),
$$

is a map of Hochschild complexes.

Also since the shuffle map

$$
S h: C^{n}(H, M) \otimes C^{n}(H, M) \longrightarrow(C(H, M) \otimes C(H, M))^{n},
$$

is a map of Hochschild complexes, the composition Sh $\rho$ is a map of Hochschild complexes and therefore it induces the map $(S h \rho)^{*}$ on the level of cohomology. One notes that in this case the shuffle map $S h$ is giving us the Künneth morphism. 
Proposition 5.3 In the Connes-Moscovici case with $M={ }^{1} k_{\delta}$, we have an explicit formula for Sh $\rho$ :

$$
\begin{aligned}
S h_{n} \rho_{n}\left(h_{1} \otimes \ldots \otimes h_{n}\right)= & \sum_{\sigma} \operatorname{sign}(\sigma)\left(h_{\sigma(1)} \otimes \ldots \otimes h_{\sigma(p)}\right) \\
& \otimes\left(h_{\sigma(p+1)} \otimes \ldots \otimes h_{\sigma(p+q)}\right),
\end{aligned}
$$

where $\sigma$ runs over all $(p, q)$-shuffles.

Proof Using $\epsilon\left(h^{(1)}\right) h^{(2)}=\epsilon\left(h^{(2)}\right) h^{(1)}=h$, we have:

$$
\begin{aligned}
& S h_{n} \Omega_{n} \Phi_{n}\left(h_{1} \otimes \ldots \otimes h_{n}\right) S h_{n}\left(\left(h_{1}^{(1)} \otimes \ldots \otimes h_{n}^{(1)}\right) \otimes\left(h_{1}^{(2)} \otimes \ldots \otimes h_{n}^{(2)}\right)\right) \\
&= \sum_{\sigma} \operatorname{sign}(\sigma) \epsilon\left(h_{\sigma(1)}^{(2)}\right) \ldots \epsilon\left(h_{\sigma(p)}^{(2)}\right) \epsilon\left(h_{\sigma(p+1)}^{(1)}\right) \ldots \epsilon\left(h_{\sigma(n)}^{(1)}\right)\left(h_{\sigma(1)}^{(1)} \otimes \ldots h_{\sigma(p)}^{(1)}\right) \\
& \otimes\left(h_{\sigma(p+1)}^{(2)} \otimes \ldots h_{\sigma(n)}^{(2)}\right) \\
&= \sum_{\sigma} \operatorname{sign}(\sigma)\left(\epsilon\left(h_{\sigma(1)}^{(2)}\right) h_{\sigma(1)}^{(1)} \otimes \ldots \otimes \epsilon\left(h_{\sigma(p)}^{(2)}\right) h_{\sigma(p)}^{(1)}\right) \otimes\left(\epsilon\left(h_{\sigma(p+1)}^{(1)}\right) h_{\sigma(p+1)}^{(2)}\right. \\
&\left.\otimes \ldots \otimes \epsilon\left(h_{\sigma(n)}^{(1)}\right) h_{\sigma(n)}^{(2)}\right) \\
&= \sum_{\sigma} \operatorname{sign}(\sigma)\left(h_{\sigma(1)} \otimes \ldots \otimes h_{\sigma(p)}\right) \otimes\left(h_{\sigma(p+1)} \otimes \ldots \otimes h_{\sigma(n)}\right) .
\end{aligned}
$$

One knows that Hochschild homology of a commutative algebra is a graded commutative and associative algebra [24]. Analogous to this classic result we have:

Proposition 5.4 The Hopf Hochschild cohomology of a cocommutative Hopf algebra $\mathcal{H}$ with coefficients in a SAYD module $M$ equipped with map $\psi$ satisfying (5.1), is a graded cocommutative and coassociative coalgebra by (5.3).

Proof The cocommutativity and coassociativity can be verified by series of long, but straightforward, computation which we omit here.

Theorem 5.1 Suppose $\mathcal{H}$ is a cocommutative Hopf algebra and $M$ a SAYD module over $\mathcal{H}$, equipped with a map $\psi: M \longrightarrow M \otimes M$ satisfying (5.1). The following map

$$
\sqcup=\mathcal{I}(\widetilde{S h} \varrho)^{*}: H C^{n}(\mathcal{H}, M) \longrightarrow \bigoplus_{p+q=n} H C^{p}(\mathcal{H}, M) \otimes H C^{q}(\mathcal{H}, M),
$$

defines a coproduct for Hopf cyclic cohomology of $\mathcal{H}$ with coefficients in $M$, where $\varrho_{n}=\oplus_{i \geq 0}\left(\Omega_{n-2 i} \Phi_{n-2 i}\right)$, and $\mathcal{I}$ is defined in (3.4).

Proof The map $(\widetilde{S h} \varrho)^{*}$ is induced by composition of the following maps of complexes,

$$
\begin{aligned}
\operatorname{Tot}^{n} B(C(H, M)) \stackrel{\varrho_{n}}{\longrightarrow} \operatorname{Tot}^{n} B(C(H, M) \times C(H, M)) \\
\stackrel{\widetilde{S h_{n}}}{\longrightarrow} \operatorname{Tot}^{n} B(C(H, M) \otimes C(H, M))
\end{aligned}
$$


Since $H$ is cocommutative the maps $\Phi_{n-2 i}$ are morphisms of cocyclic modules by Proposition 5.1. Since $\Omega_{n-2 i}$ are maps of cocyclic modules by Lemma 4.2 , the morphism $\varrho_{n}$ is a map of cocyclic modules. Therefore the composition $\widetilde{S h} \varrho$ is a map of cocyclic modules which induces the map $(\widetilde{S h} \varrho)^{*}$ on the level of cyclic cohomology. Now we compose this map by Künneth map $\mathcal{I}$ defined in (3.4) to obtain the coproduct $\sqcup$ on the level of cyclic cohomology.

Theorem 5.2 Suppose $\mathcal{H}$ is a cocommutative Hopf algebra and $M$ a SAYD module over $\mathcal{H}$, equipped with a map $\psi: M \longrightarrow M \otimes M$ satisfying (5.1). The following map

$$
\sqcup=\nabla(\widetilde{S h} \varrho)^{*}: H P^{n}(\mathcal{H}, M) \longrightarrow \bigoplus_{i+j=n} H P^{i}(\mathcal{H}, M) \otimes H P^{j}(\mathcal{H}, M), \quad n=0,1,
$$

defines a coproduct for periodic Hopf cyclic cohomology where $\bar{\varrho}=\bigoplus_{i \geq 0} \Phi_{2 i+*}$, and $\nabla$ is as (4.7).

Now we provide some examples of the map $\psi$ satisfying the condition (5.1).

Example 5.1 Let $\mathcal{H}=k G$ be the group algebra of the discrete group $G$. Suppose $M$ is a SAYD module over $\mathcal{H}$. One can check that $M$ is a $G$-graded vector space $M=\oplus_{g \epsilon G} M_{g}$, where the coaction $\boldsymbol{\nabla}$ is defined by $\boldsymbol{\nabla}(m)=g \otimes m$. The stability condition implies $g m=m$ for all $m \in M_{g}$, and anti-Yetter-Drinfeld condition is equivalent to $h m \in M_{h g h^{-1}}$ for all $g$ and $h \in G$. Since $g$ is a group-like element, any linear map $\psi: M \longrightarrow M \otimes M$, with $\psi\left(M_{g}\right) \subseteq M_{g} \otimes M_{g}$ satisfies the condition (5.1).

Example 5.2 Let $\mathcal{H}=\mathcal{U}(\mathfrak{g})$ be the universal enveloping algebra of a Lie algebra $\mathfrak{g}$ and $M$ an arbitrary module over $\mathcal{H}$. We can define a comodule structure on $M$ by trivial coaction: $m \longmapsto 1 \otimes m$. It can be shown that $M$ is a SAYD module over $\mathcal{H}$ and any linear map $\psi: M \longrightarrow M \otimes M$, satisfies (5.1).

Example 5.3 Let $\mathcal{H}$ be any cocommutative Hopf algebra and $M={ }^{1} k_{\delta}$. It is easy to check that any linear map $\psi: M \longrightarrow M \otimes M$, satisfies the condition (5.1). We use this example to get a coproduct in Connes-Moscovici setting in the following corollary.

Corollary 5.1 If $M={ }^{1} k_{\delta}$ then the coproducts (5.3), (5.5), (5.6) reduce to coproducts for Connes-Moscovici Hopf Hochschild, cyclic and periodic cyclic cohomology of a cocommutative Hopf algebra $\mathcal{H}$.

\section{Relation with coproduct in Lie algebra homology}

In this section we show that the coproduct (5.6) for periodic Hopf cyclic cohomology for $\mathcal{H}=\mathcal{U}(\mathfrak{g})$, the universal enveloping algebra of a Lie algebra $\mathfrak{g}$, agrees with the coproduct in Lie algebra homology. Recall that the periodic Hopf cyclic cohomology of $\mathcal{H}=\mathcal{U}(\mathfrak{g})$ is given by [3]

$$
H P_{(\delta, 1)}^{*}(\mathcal{U}(\mathfrak{g})) \cong \bigoplus_{k \geq 0} H_{2 k+*}\left(\mathfrak{g}, k_{\delta}\right)
$$


where $*=0,1$, and $k_{\delta}$ is a $\mathfrak{g}$-module via the character $\delta$. The right hand side of this isomorphism is the Lie algebra homology of $\mathfrak{g}$ with coefficients in $k_{\delta}$. It is the homology of the the following mixed complex:

$$
\bigwedge \mathfrak{g} \underset{0}{\stackrel{d_{\text {Lie }}}{\leftrightarrows}} \bigwedge \mathfrak{g} \underset{0}{\stackrel{d_{\text {Lie }}}{\leftrightarrows}} \bigwedge^{2} \mathfrak{g} \underset{0}{\stackrel{d_{\text {Lie }}}{\leftrightarrows}} \ldots
$$

where $d_{\text {Lie }}$ denotes the Chevalley-Eilenberg differential for Lie algebra homology. The isomorphism (6.1) is induced by the anti symmetrization map $A_{n}: \bigwedge^{n} \mathfrak{g} \longrightarrow$ $\mathcal{U}(\mathfrak{g})^{\otimes n}$, given by

$$
\left(g_{1} \wedge \cdots \wedge g_{n}\right) \longmapsto \frac{1}{n !}\left(\sum_{\sigma} \operatorname{sign}(\sigma)\left(g_{\sigma(1)} \otimes \cdots \otimes g_{\sigma(n)}\right) .\right.
$$

Here $\sigma$ runs over all permutations of the set $\{1,2, \ldots, n\}$. If $\delta=\varepsilon$, then $d_{\text {Lie }}$ is given by

$$
d_{\text {Lie }}\left(g_{1} \wedge \cdots \wedge g_{n}\right)=\sum_{1 \leq i \leq i \leq n}(-1)^{i+j+1}\left[g_{i}, g_{j}\right] \wedge\left(g_{1} \wedge \cdots \wedge \hat{g}_{i} \wedge \cdots \wedge \hat{g_{j}} \wedge \cdots \wedge g_{n}\right)
$$

Let $C_{n}^{\text {Lie }}(\mathfrak{g})=\bigwedge^{n} \mathfrak{g}$. One knows that Lie algebra homology with trivial coefficients is a coalgebra by the following coproduct

$$
\cup_{\text {Lie }}: C_{n}^{\text {Lie }}(\mathfrak{g}) \longrightarrow \bigoplus_{p+q=n}\left(C_{p}^{\text {Lie }}(\mathfrak{g})\right) \otimes\left(C_{q}^{\text {Lie }}(\mathfrak{g})\right)
$$

given by

$$
\cup_{\text {Lie }}\left(g_{1} \wedge \cdots \wedge g_{n}\right)=\sum_{\sigma} \operatorname{sign}(\sigma)\left(g_{\sigma(1)} \wedge \cdots \wedge g_{\sigma(p)}\right) \otimes\left(g_{\sigma(p+1)} \wedge \ldots \wedge g_{\sigma(n)}\right),
$$

where $\sigma$ runs over all $(p, q)$-shuffles. Since $\varepsilon\left(g_{i}\right)=0$ for all $g_{i} \in \mathfrak{g}$, the image of the anti symmetrization map is in the normalized complex of $C^{n}(\mathcal{U}(\mathfrak{g}))=\mathcal{U}(\mathfrak{g})^{\otimes n}$. One can define the following map of mixed complexes:

$$
\mathbb{A}_{n}=\sum_{i+j=n} A_{i} \otimes A_{j}: \bigoplus_{i+j=n}\left(\Lambda^{i} \mathfrak{g}\right) \otimes\left(\Lambda^{j} \mathfrak{g}\right) \longrightarrow \bigoplus_{i+j=n}\left(\mathcal{U}^{i}(\mathfrak{g})\right) \otimes\left(\mathcal{U}^{j}(\mathfrak{g})\right)
$$

It induces a map:

$$
\left.\left.\mathbb{A}^{*}: \bigoplus_{k \geq 0} H_{2 k+*}\left(C^{L i e}(\mathfrak{g})\right) \otimes C^{L i e}(\mathfrak{g})\right)\right) \longrightarrow H P^{*}(C(\mathcal{U}(\mathfrak{g}) \otimes C(\mathcal{U}(\mathfrak{g}))
$$


Now using the Künneth formula (4.7), we obtain the following map:

$$
\begin{aligned}
\nabla\left(\mathbb{A} \cup_{L i e}\right)^{*}: \bigoplus_{n \geq 0} H_{2 n+1}\left(\mathfrak{g}, k_{\varepsilon}\right) \longrightarrow & H P_{(\varepsilon, 1)}^{1}(\mathcal{U}(\mathfrak{g})) \otimes H P_{(\varepsilon, 1)}^{0}(\mathcal{U}(\mathfrak{g})) \\
& \oplus H P_{(\varepsilon, 1)}^{0}(\mathcal{U}(\mathfrak{g})) \otimes H P_{(\varepsilon, 1)}^{1}(\mathcal{U}(\mathfrak{g}))
\end{aligned}
$$

and similarly for the even case.

Theorem 6.1 Under the isomorphism (6.1), the coproduct (5.6) for the periodic cyclic cohomology of the universal enveloping algebra $\mathcal{U}(\mathfrak{g})$ with trivial coefficients coincides with the coproduct of Lie algebra homology. Equivalently, the following diagram commutes on the level of homology.

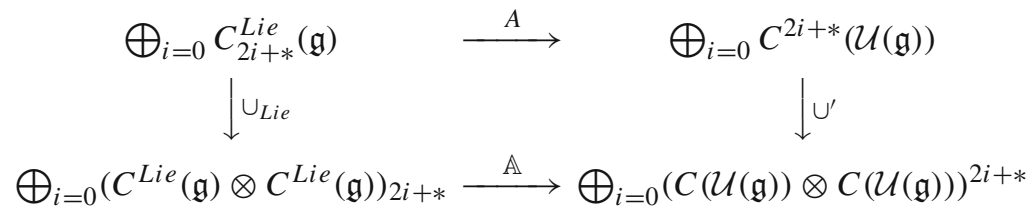

where $\cup^{\prime}=\widetilde{S h} \Omega \Delta^{\otimes n}$.

Proof The commutativity of the diagram is equivalent to

$$
s h_{n} \Omega_{n} \Delta_{\mathcal{U}(\mathfrak{g})}^{\otimes n} A_{n} \oplus s h_{n+2}^{\prime} \Omega_{n+2} \Delta_{\mathcal{U}(\mathfrak{g})}^{\otimes n+2} A_{n+2}=\mathbb{A}_{n} \cup_{\text {Lie }}, \quad n \in \mathbb{N} .
$$

Using the Proposition 5.3, one can easily see

$$
s h_{n} \Omega_{n} \Delta_{\mathcal{U}(\mathfrak{g})}^{\otimes n} A_{n}=\mathbb{A}_{n} \cup_{\text {Lie }},
$$

where

$$
\begin{aligned}
\mathbb{A}_{n} \cup_{\text {Lie }}\left(g_{1} \wedge \cdots \wedge g_{n}\right)= & \sum_{p=0}^{n} \sum_{\sigma \in S_{n}} \operatorname{sign}(\sigma)\left(g_{\sigma(1)} \otimes \cdots \otimes g_{\sigma(p)}\right) \\
& \otimes\left(g_{\sigma(p+1)} \otimes \cdots \otimes g_{\sigma(n)}\right) .
\end{aligned}
$$

Now it is enough to show:

$$
s h_{n+2}^{\prime} \Omega_{n+2} \Delta_{U(\mathfrak{g})}^{\otimes n+2} A_{n+2}=A_{n} d_{\text {Lie }},
$$

which means this term will be zero on the level of homology. To do this, first we compute $\Omega_{n+2} \Delta_{\mathcal{U}(\mathfrak{g})}^{\otimes(n+2)} A_{n+2}$ :

$$
\begin{aligned}
& \Omega_{n+2}\left(\Delta_{\mathcal{U}(\mathfrak{g})}^{\otimes(n+2)}\right) A_{n+2}\left(g_{1} \wedge \cdots \wedge g_{n+2}\right)=\Omega_{n+2} \sum_{\sigma \in S_{n+2}} \Delta_{\mathcal{U}(\mathfrak{g})}^{\otimes(n+2)}\left(g_{\sigma(1)} \otimes \ldots \otimes g_{\sigma(n+2)}\right) \\
& =\Omega_{n+2} \sum_{\sigma \in S_{n+2}}\left(\Delta\left(g_{\sigma(1)}\right) \Delta\left(g_{\sigma(2)}\right) \ldots \Delta\left(g_{\sigma(n+2)}\right)\right)
\end{aligned}
$$




$$
\begin{aligned}
= & \Omega_{n+2} \sum_{\sigma \in S_{n+2}}\left(\left(1 \otimes g_{\sigma(1)}+g_{\sigma(1)} \otimes 1\right) \ldots\left(1 \otimes g_{\sigma(n+2)}+g_{\sigma(n+2)} \otimes 1\right)\right) \\
= & \sum_{p=0}^{n+2} \sum_{\sigma \in S_{n+2}}\left(g_{\sigma(1)} \otimes \cdots \otimes g_{\sigma(p)} \otimes 1 \otimes \cdots \otimes 1\right) \\
& \otimes\left(g_{\sigma(p+1)} \otimes \cdots \otimes g_{\sigma(n+2)} \otimes 1 \otimes \cdots \otimes 1\right)
\end{aligned}
$$

where in the last sum, $g_{\sigma(1)}, \ldots, g_{\sigma(p)}$ and $g_{\sigma(p+1)}, \ldots, g_{\sigma(p+q)}$ appear in $(p, q)$ shuffle spots. For example when $n=1$, we have:

$$
\begin{aligned}
\Omega_{3} \Delta_{\mathcal{U}(\mathfrak{g})}^{\otimes 3} A_{3}\left(g_{1} \wedge g_{2} \wedge g_{3}\right)= & (1 \otimes 1 \otimes 1) \otimes\left(g_{\sigma(1)} \otimes g_{\sigma(2)} \otimes g_{\sigma(3)}\right) \\
& +\left(1 \otimes 1 \otimes g_{\sigma(3)}\right) \otimes\left(g_{\sigma(1)} \otimes g_{\sigma(2)} \otimes 1\right) \\
& +\left(1 \otimes g_{\sigma(2)} \otimes 1\right) \otimes\left(g_{\sigma(1)} \otimes 1 \otimes g_{\sigma(3)}\right) \\
& +\left(1 \otimes g_{\sigma(2)} \otimes g_{\sigma(3)}\right) \otimes\left(g_{\sigma(1)} \otimes 1 \otimes 1\right) \\
& +\left(g_{\sigma(1)} \otimes g_{\sigma(2)} \otimes 1\right) \otimes\left(1 \otimes 1 \otimes g_{\sigma(3)}\right) \\
& +\left(g_{\sigma(1)} \otimes g_{\sigma(2)} \otimes g_{\sigma(3)}\right) \otimes(1 \otimes 1 \otimes 1) \\
& +\left(g_{\sigma(1)} \otimes 1 \otimes 1\right) \otimes\left(1 \otimes g_{\sigma(2)} \otimes g_{\sigma(3)}\right) \\
& +\left(g_{\sigma(1)} \otimes 1 \otimes g_{\sigma(3)}\right) \otimes\left(1 \otimes g_{\sigma(2)} \otimes 1\right)
\end{aligned}
$$

Since $s h_{n}^{\prime}=\oplus_{i+j=n+2} s h_{i, j}^{\prime}$, it is enough to show $s h_{i, j}^{\prime}=0$, for all $1 \leq i, j \leq n+1$. For $s h_{i, j}^{\prime}$, we apply degeneracies, i.e., counit $\varepsilon, n+2$ times on the following elements:

$$
\left(g_{\sigma(1)} \otimes \cdots \otimes g_{\sigma(p)} \otimes 1 \otimes \cdots \otimes 1\right) \otimes\left(g_{\sigma(p+1)} \otimes \cdots \otimes g_{\sigma(n+2)} \otimes 1 \otimes \cdots \otimes 1\right),
$$

and then we apply $\left(s_{\sigma^{\prime}(1)} \otimes \ldots s_{\left.\sigma^{\prime}(q)\right)} \otimes\left(s_{\sigma^{\prime}(q+1)} \ldots s_{\sigma^{\prime}(n)}\right)\right.$. Now for the terms which are zero, there is nothing remained to prove. Those which are not zero, should be in the forms of

$$
\left(g_{\sigma(1)} \otimes \cdots \otimes g_{\sigma(i)}\right) \otimes\left(g_{\sigma(i+1)} \otimes \cdots \otimes g_{\sigma(n+2)}\right),
$$

for some $1 \leq i \leq n+2$. Here $\sigma$ runs over all permutations in $S_{n+2}$, the symmetric group with $n+2$ terms. Now we compute

$$
\sigma_{i-p-1}^{i-1} \tau^{p+1}\left(g_{\sigma(1)} \otimes \cdots \otimes g_{\sigma(i)}\right) \otimes \sigma_{j-q-1}^{j-1} \tau^{q+1}\left(g_{\sigma(i+1)} \otimes \cdots \otimes g_{\sigma(n+2)}\right) .
$$

Let $\sigma(i+1), \ldots, \sigma(n)$ be fixed. For any $0 \leq k \leq n+2$, we have:

$$
\begin{aligned}
& \sigma_{i-k}^{i-1} \tau^{k}\left(g_{\sigma(1)} \otimes \cdots \otimes g_{\sigma(i)}\right) \\
& =\sigma_{i-k}^{i-1}\left(\sum_{\sigma} S\left(g_{\sigma(k)}^{(i)}\right) g_{\sigma(k+1)} \otimes S\left(g_{\sigma(k)}^{(i-1)}\right) g_{\sigma(k+2)} \otimes \cdots \otimes S\left(g_{\sigma(k)}^{(k)}\right) \sigma\right. \\
& \left.\otimes S\left(g_{\sigma(k)}^{(k-1)}\right) g_{\sigma(1)} \otimes \cdots \otimes S\left(g_{\sigma(k)}^{(2)}\right) g_{\sigma(2)} \otimes \widetilde{S}\left(g_{\sigma(k)}^{(1)}\right) \sigma g_{\sigma(i-1)}\right)
\end{aligned}
$$




$$
\begin{aligned}
& =\sigma_{i-k}^{i-1}\left(\sum_{\sigma \in S_{p}} \sum_{r=k+1}^{i-1} g_{\sigma(k+1)} \otimes \ldots g_{\sigma(r-1)}\right. \\
& \otimes g_{\sigma(k)} g_{\sigma(r)} \otimes g_{\sigma(r+1)} \otimes \cdots \otimes g_{\sigma(i)} \otimes 1 \otimes g_{\sigma(1)} \otimes \cdots \otimes g_{\sigma(i-1)} \\
& \left.+g_{\sigma(k+1)} \otimes \cdots \otimes g_{\sigma(i)} \otimes g_{\sigma(k)} \otimes g_{\sigma(1)} \otimes \cdots \otimes g_{\sigma(i-1)}\right) \\
& =\sum_{\sigma \in S_{i}} \sum_{r=k+1}^{p-1} g_{\sigma(k+1)} \otimes \cdots \otimes g_{\sigma(r-1)} \\
& \otimes g_{\sigma(k)} g_{\sigma(r)} \otimes g_{\sigma(r+1)} \otimes \cdots \otimes g_{\sigma(i)} \otimes g_{\sigma(1)} \otimes \cdots \otimes g_{\sigma(i-1)} \\
& =\sum_{\sigma \in S_{i}, \sigma(v)>\sigma(w)} g_{\sigma(1)} \otimes \cdots \otimes g_{\sigma(v)} g_{\sigma(w)} \otimes \cdots \otimes g_{\sigma(i)} \\
& +\sum_{\sigma \in S_{i}, \sigma(v)>\sigma(w)} g_{\sigma(1)} \otimes \cdots \otimes g_{\sigma(w)} g_{\sigma(v)} \otimes \cdots \otimes g_{\sigma(i)} \\
& =\sum_{\sigma \in S_{i}, \sigma(v)>\sigma(w)} g_{\sigma(1)} \otimes \cdots \otimes g_{\sigma(v)} g_{\sigma(w)}-g_{\sigma(w)} g_{\sigma(v)} \otimes \cdots \otimes g_{\sigma(i)} \\
& =\sum_{\sigma \in S_{i}, \sigma(v)>\sigma(w)} g_{\sigma(1)} \otimes \cdots \otimes\left[g_{\sigma(v)}, g_{\sigma(w)}\right] \otimes \cdots \otimes g_{\sigma(i)} \\
& =A\left(\left[g_{\sigma(v)}, g_{\sigma(w)}\right] \wedge g_{\sigma(1)} \wedge \cdots \wedge \hat{g}_{\sigma(w)} \wedge \cdots \wedge \hat{g}_{\sigma(v)} \wedge \cdots \wedge g_{\sigma(i)}\right) \\
& =A d^{\text {Lie }}\left(g_{1} \wedge \cdots \wedge g_{i}\right) \text {. }
\end{aligned}
$$

\section{Coproducts in Hopf cyclic homology}

In this section we define coproducts for Hopf cyclic homology in the sense of [12,20]. We show that for group algebras our coproduct coincides with the coproduct in group homology. In this section we assume $M$ is a left-left stable anti-Yetter-Drinfeld module on a Hopf algebra $\mathcal{H}$. Let

$$
\widetilde{C}_{n}(\mathcal{H}, M)=\mathcal{H}^{\otimes n+1} \square_{\mathcal{H}} M \quad n \geq 0,
$$

where $X \square_{\mathcal{H}} Y=\operatorname{ker}\left(\Delta_{X} \otimes i d-i d \otimes{ }_{Y} \Delta\right): X \otimes Y \longrightarrow X \otimes \mathcal{H} \otimes Y$ is the cotensor product of $X$ and $Y[12,20]$. One can define faces, degeneracies and cyclic maps on $\left\{\widetilde{C}_{n}(\mathcal{H}, M)\right\}_{n \in \mathbb{N}}$ as follows:

$$
\begin{aligned}
& \delta_{i}\left(h_{0} \otimes \ldots \otimes h_{n} \otimes m\right)=h_{0} \otimes \ldots \otimes h_{i} h_{i+1} \otimes \ldots \otimes h_{n} \otimes m, \quad 0 \leq i<n, \\
& \delta_{n}\left(h_{0} \otimes \ldots \otimes h_{n} \otimes m\right)=h_{n}^{(0)} h_{0} \otimes h_{1} \ldots \otimes h_{n-1} \otimes h_{n}^{(1)} m, \\
& \sigma_{i}\left(h_{0} \otimes \ldots \otimes h_{n} \otimes m\right)=h_{0} \otimes \ldots \otimes h_{i} \otimes 1 \otimes \ldots \otimes h_{n} \otimes m, \quad 0 \leq i \leq n, \\
& \tau_{n}\left(h_{0} \otimes \ldots \otimes h_{n} \otimes m\right)=h_{n}^{(0)} \otimes h_{0} \otimes \ldots \otimes h_{n-1} \otimes h_{n}^{(1)} m .
\end{aligned}
$$


The homology of the above cyclic module is by definition the Hopf cyclic homology of the Hopf algebra $\mathcal{H}$ with coefficients in the SAYD module $M$ and will be denoted by $\widetilde{H C_{*}}(\mathcal{H}, M)$.

Lemma 7.1 Let $\mathcal{H}$ and $\mathcal{K}$ be two Hopf algebras and $M$ and $N$ SAYD modules over $\mathcal{H}$ and $\mathcal{K}$ respectively. The following map is an isomorphism of cyclic modules

$$
\Omega_{n}:(\mathcal{H} \otimes \mathcal{K})^{\otimes n+1} \square_{\mathcal{H} \otimes \mathcal{K}}(M \otimes N) \longrightarrow\left(\mathcal{H}^{\otimes n+1} \square_{\mathcal{H}} M\right) \otimes\left(\mathcal{K}^{\otimes n+1} \square_{\mathcal{K}} N\right),
$$

given by

$$
\begin{aligned}
& \left(\left(h_{0} \otimes k_{0}\right) \otimes \cdots \otimes\left(h_{n} \otimes k_{n}\right) \otimes(m \otimes r)\right) \\
& \quad \longmapsto\left(h_{0} \otimes \cdots \otimes h_{n} \otimes m\right) \otimes\left(k_{0} \otimes \cdots \otimes k_{n} \otimes r\right) .
\end{aligned}
$$

Proof We prove $\Omega_{n}$ commutes with $\delta_{i}$ and $\tau_{n}$, where $0 \leq i<n$. One can easily verify this for $\delta_{n}$ and degeneracies.

$$
\begin{aligned}
\left(\delta_{i}\right. & \left.\otimes \delta_{i}\right) \Omega_{n}\left(\left(h_{0} \otimes k_{0}\right) \otimes \cdots \otimes\left(h_{n} \otimes k_{n}\right) \otimes(m \otimes r)\right) \\
& =\left(\delta_{i} \otimes \delta_{i}\right)\left(\left(h_{0} \otimes \cdots \otimes h_{n} \otimes m\right),\left(k_{0} \otimes \cdots \otimes k_{n} \otimes r\right)\right) \\
& =\delta_{i}\left(h_{0} \otimes \cdots \otimes h_{n} \otimes m\right) \otimes \delta_{i}\left(k_{0} \otimes \cdots \otimes k_{n} \otimes r\right) \\
& =\left(h_{0} \otimes \ldots h_{i} h_{i+1} \otimes \cdots \otimes h_{n} \otimes m\right) \otimes\left(k_{0} \otimes \ldots k_{i} k_{i+1} \otimes \cdots \otimes k_{n} \otimes r\right) \\
& =\Omega_{n}\left(( h _ { 0 } \otimes k _ { 0 } ) \otimes \cdots \otimes ( h _ { i } h _ { i + 1 } \otimes k _ { i } k _ { i + 1 } ) \otimes \cdots \otimes \left(\left(h_{n} \otimes k_{n}\right)\right.\right. \\
& =\Omega_{n} \delta_{i}\left(\left(h_{0} \otimes k_{0}\right) \otimes \cdots \otimes\left(h_{n} \otimes k_{n}\right) \otimes(m \otimes r)\right) .
\end{aligned}
$$

Since $\mathcal{H}$ is an $\mathcal{H}$-comodule algebra by comultiplication, we have:

$$
\begin{aligned}
& \left(\tau_{n} \otimes \tau_{n}\right) \Omega_{n}\left(\left(h_{0} \otimes k_{0}\right) \otimes \cdots \otimes\left(h_{n} \otimes k_{n}\right) \otimes(m \otimes r)\right) \\
& \quad=\tau_{n}\left(h_{0} \otimes \cdots \otimes h_{n} \otimes m\right) \otimes \tau_{n}\left(k_{0} \otimes \cdots \otimes k_{n} \otimes r\right) \\
& \quad=\left(h_{n}^{(1)} \otimes h_{0} \otimes \cdots \otimes h_{n-1} \otimes h_{n}^{(2)} m\right) \otimes\left(k_{n}^{(1)} \otimes k_{0} \otimes \cdots \otimes k_{n-1} \otimes k_{n}^{(2)} r\right) . \\
& =\Omega_{n}\left(h_{n}^{(1)} \otimes k_{n}^{(1)}\right) \otimes\left(h_{0} \otimes k_{0}\right) \otimes \cdots \otimes\left(h_{n-1} \otimes h_{n-1}\right) \otimes\left(h_{n}^{(2)} m \otimes k_{n}^{(2)} r\right) \\
& =\Omega_{n}\left(h_{n}^{(1)} \otimes k_{n}^{(1)}\right) \otimes\left(h_{0} \otimes k_{0}\right) \otimes \cdots \otimes\left(h_{n-1} \otimes h_{n-1}\right) \otimes\left(h_{n}^{(2)} m \otimes k_{n}^{(2)} r\right) \\
& =\Omega_{n} \tau_{n}\left(\left(h_{0} \otimes k_{0}\right) \otimes \ldots\left(h_{n} \otimes k_{n}\right) \otimes(m \otimes r)\right) .
\end{aligned}
$$

Using the previous lemma, the Eilenberg-Zilber isomorphism, and the Künneth formula we obtain the following proposition:

Proposition 7.1 Let $\mathcal{H}$ and $\mathcal{K}$ be two Hopf algebras and $M$ and $N$ be SAYD modules over $\mathcal{H}$ and $\mathcal{K}$, respectively. We have the following isomorphism for Hopf Hochschild homology with coefficients

$$
\widetilde{H H}_{n}(\mathcal{H} \otimes \mathcal{K}, M \otimes N) \simeq \bigoplus_{i+j=n} \widetilde{H H}_{i}(\mathcal{H}, M) \otimes \widetilde{H H}_{j}(\mathcal{K}, N)
$$


Also one has the following long exact sequence for Hopf cyclic homology with coefficients:

$$
\begin{aligned}
& \ldots \longrightarrow \widetilde{H C_{n}}(\mathcal{H} \otimes \mathcal{K}, M \otimes N) \stackrel{\mathcal{I}}{\longrightarrow} \bigoplus_{i+j=n} \widetilde{H C_{i}}(\mathcal{H}, M) \otimes \widetilde{H C}(\mathcal{K}, N) \stackrel{S \otimes i d-i d \otimes S}{\longrightarrow} \\
& \bigoplus_{i+j=n-2} \widetilde{H C_{i}}(\mathcal{H}, M) \otimes \widetilde{H C}(\mathcal{K}, N) \stackrel{\partial}{\longrightarrow} \widetilde{H C_{n-1}}(\mathcal{H} \otimes \mathcal{K}, M \otimes N) \longrightarrow \ldots
\end{aligned}
$$

Furthermore if $\widetilde{(H C}(\mathcal{H}, M)[-2 m], S)_{-2 m}$ satisfies the Mittag-Leffler condition and $\widetilde{H P} *(\mathcal{H}, M)$ is a finite dimensional vector space, then we obtain the following isomorphism for the periodic Hopf cyclic homology with coefficients:

$$
\widetilde{H P_{0}}(\mathcal{H} \otimes \mathcal{K}, M \otimes N) \simeq \widetilde{H P_{0}}(\mathcal{H}, M) \otimes \widetilde{H P_{0}}(\mathcal{K}, N) \oplus \widetilde{H P_{1}}(\mathcal{H}, M) \otimes \widetilde{H P}_{1}(\mathcal{K}, N),
$$

and similarly for the odd case.

Proposition 7.2 Let $\mathcal{H}$ be a cocommutative Hopf algebra and $M$ a SAYD module over $\mathcal{H}$ equipped with a map $\psi: M \longrightarrow M \otimes M$ satisfying the condition:

$$
\psi(h . m)=\Delta(h) . \psi(m)
$$

The following map

$$
\rho_{n}=\Omega_{n} \Phi_{n}: \widetilde{C}_{n}(\mathcal{H}, M) \longrightarrow(\widetilde{C}(\mathcal{H}, M) \times \widetilde{C}(\mathcal{H}, M))_{n},
$$

is a map of cyclic modules where

$$
\Phi_{n}=\psi \otimes \Delta^{\otimes n+1}: \widetilde{C}_{n}(\mathcal{H}, M) \longrightarrow \widetilde{C}_{n}(\mathcal{H} \otimes \mathcal{H}, M \otimes M) .
$$

Proof We only check the commutativity of $\rho_{n}$ with $\delta_{n}$. The condition (7.3) is equivalent to

$$
(h . m)_{(1)} \otimes(h . m)_{(2)}=h^{(1)} \cdot m_{(1)} \otimes h^{(2)} \cdot m_{(2)} .
$$

By cocommutativity of $\mathcal{H}$ we have:

$$
\begin{aligned}
\left(\delta_{n} \otimes \delta_{n}\right) \Omega_{n}\left(\Delta^{\otimes n+1} \otimes \psi\right)\left(h_{0} \otimes \ldots \otimes h_{n} \otimes m\right) \\
=\delta_{n}\left(h_{0}^{(1)} \otimes \cdots \otimes h_{n}^{(1)} \otimes m_{(1)}\right) \otimes \delta_{n}\left(h_{0}^{(2)} \otimes \cdots \otimes h_{n}^{(2)} \otimes m_{(2)}\right) \\
=\left(h_{n}^{(1)(1)} h_{0}^{(1)} \otimes h_{1}^{(1)} \otimes \ldots h_{n-1}^{(1)} \otimes h_{n}^{(1)(2)} m_{(1)}\right) \\
\quad \otimes\left(h_{n}^{(2)(1)} h_{0}^{(2)} \otimes h_{1}^{(2)} \otimes \ldots h_{n-1}^{(2)} \otimes h_{n}^{(2)(2)} m_{(2)}\right) \\
=\Omega_{n}\left(\left(h_{n}^{(1)(1)} h_{0}^{(1)} \otimes\left(h_{n}^{(2)(1)} h_{0}^{(2)}\right) \otimes\left(h_{1}^{(1)} \otimes h_{1}^{(2)}\right)\right.\right. \\
\quad \otimes \cdots \otimes\left(h_{n-1}^{(1)} \otimes h_{n-1}^{(2)}\right) \otimes\left(h_{n}^{(1)(2)} m_{(1)} \otimes h_{n}^{(2)(2)} m_{(2)}\right)
\end{aligned}
$$




$$
\begin{aligned}
= & \Omega_{n}\left(\left(h_{n}^{(1)(1)} h_{0}^{(1)} \otimes\left(h_{n}^{(1)(2)} h_{0}^{(2)}\right) \otimes\left(h_{1}^{(1)} \otimes h_{1}^{(2)}\right)\right.\right. \\
& \left.\otimes \cdots \otimes\left(h_{n-1}^{(1)} \otimes h_{n-1}^{(2)}\right) \otimes\left(h_{n}^{(2)} m\right)_{(1)} \otimes\left(h_{n}^{(2)} m\right)_{(2)}\right) \\
= & \Omega_{n}\left(\Delta^{\otimes n+1} \otimes \psi\right)\left(h_{n}^{(1)} h_{0} \otimes h_{1} \otimes \cdots \otimes h_{n-1} \otimes h_{n}^{(2)} m\right) \\
= & \Omega_{n}\left(\Delta^{\otimes n+1} \otimes \psi\right) \delta_{n}\left(h_{0} \otimes \cdots \otimes h_{n} \otimes m\right) .
\end{aligned}
$$

Now we are ready to define the desired coproducts.

Proposition 7.3 Let $\mathcal{H}$ be a cocommutative Hopf algebra, $M$ a SAYD module over $\mathcal{H}$ equipped with a map $\psi: M \longrightarrow M \otimes M$ satisfying (7.3). The following maps define coproducts for $\widetilde{H H}_{*}(\mathcal{H}, M), \widetilde{H C}_{*}(\mathcal{H}, M)$ and $\widetilde{H P_{*}}(\mathcal{H}, M)$ :

$$
\sqcup=\mathfrak{I}\left(A W_{n} \Omega_{n} \Phi_{n}\right)^{*}: \widetilde{H H}_{n}(\mathcal{H}, M) \longrightarrow \bigoplus_{p+q=n} \widetilde{H H}_{p}(\mathcal{H}, M) \otimes \widetilde{H H}_{q}(\mathcal{H}, M),
$$

and

$$
\sqcup=\mathcal{I}\left(\widetilde{A W}_{n} \bar{\Omega}_{n} \bar{\Phi}_{n}\right)^{*}: \widetilde{H C_{n}}(\mathcal{H}, M) \longrightarrow \bigoplus_{p+q=n} \widetilde{H C}_{p}(\mathcal{H}, M) \otimes \widetilde{H C_{q}}(\mathcal{H}, M)
$$

where $\bar{\Phi}_{n}=\oplus_{i \geq 0} \Phi_{n-2 i}$ and $\bar{\Omega}_{n}=\oplus_{i \geq 0} \Omega_{n-2 i}$. Also if $\left.\widetilde{(H C}(H, M)[-2 m], S\right)_{-2 m}$ satisfies the Mittag-Leffler condition and $\widetilde{H P}(\mathcal{H}, M)$ is a finite dimensional vector space, then

$$
\begin{aligned}
\sqcup= & \left.\nabla \widetilde{\left(A W_{n}\right.} \underline{\Omega}_{n} \underline{\Phi}_{n}\right)^{*}: \widetilde{H P_{1}}(\mathcal{H}, M) \longrightarrow \widetilde{H P_{0}}(\mathcal{H}, M) \\
& \otimes \widetilde{H P}_{1}(\mathcal{H}, M) \oplus \widetilde{H P_{1}}(\mathcal{H}, M) \otimes \widetilde{H P_{0}}(\mathcal{H}, M),
\end{aligned}
$$

where $\underline{\Phi}_{n}=\oplus_{i \geq 0} \Phi_{2 i}$ and $\underline{\Omega}_{n}=\oplus_{i \geq 0} \Omega_{2 i}$ and similarly for the even case.

Proof These are the results of the fact that by Lemma 7.1, the morphisms $\Phi, \bar{\Phi}$ and $\Phi$ are maps of $(b, B)$-mixed complexes.

Recall that the Hopf Hochschild and Hopf cyclic homology of a group algebra $\mathcal{H}=k G$ are computed in [20] and are given by

$$
\widetilde{H H}_{n}(k G) \cong H_{n}(G, k),
$$

and

$$
\widetilde{H C_{n}}(k G) \stackrel{\theta}{\cong} \bigoplus_{i \geq 0} H_{n-2 i}(G, k)
$$


where on the right hand side group homologies of $G$ with trivial coefficients appear. The coproduct in group homology is induced by the map

$$
\sqcup_{G r}\left(g_{1}, \ldots, g_{n}\right)=\sum_{k=0}^{n}\left(g_{1} \otimes \cdots \otimes g_{k}\right) \otimes\left(g_{k+1} \otimes \cdots \otimes g_{n}\right) .
$$

Lemma 7.2 The coproduct for Hopf Hochschild homology of a group algebra $k G$ coincides with the coproduct of group homology, i.e.,

$$
\sqcup\left(g_{1} \otimes \cdots \otimes g_{n}\right)=\sqcup_{G r}\left(g_{1}, \ldots, g_{n}\right) .
$$

Theorem 7.1 Under the isomorphism (7.8), the coproduct (7.5) for the Hopf cyclic homology of the group algebra $k G$ with trivial coefficients coincides with the coproduct of group homology. Equivalently, the following diagram commutes on the level of homology.

$$
\begin{array}{ccc}
\widetilde{C_{n}}(k G) & \stackrel{\theta_{n}}{\longrightarrow} & C_{n}^{G r}(G, k) \\
\downarrow & & \downarrow \cup_{G r}^{\prime} \\
(\widetilde{C}(k G) \otimes \widetilde{C}(k G))_{n} \stackrel{\theta^{\prime}}{\longrightarrow}\left(C^{G r}(G, k) \otimes C^{G r}(G, k)\right)_{n}
\end{array}
$$

where $\cup^{\prime}=\widetilde{A W} \Omega \Delta^{\otimes n}, \widetilde{C_{n}}(k G)=k G^{n}$ and $\theta^{\prime}=\sum_{i \geq 0} \theta_{n-2 i} \otimes \theta_{n-2 i}$

\section{Cup products in Hopf cyclic homology}

Dual to previous coproducts, in this section we define cup products for Hopf Hochschild and Hopf cyclic homology of a commutative Hopf algebra.

Let $\mathcal{H}$ and $\mathcal{K}$ be two Hopf algebras. The map

$$
\times=\operatorname{sh}_{p, q}: \widetilde{C}_{p}(\mathcal{H}) \otimes \widetilde{C}_{q}(\mathcal{K}) \longrightarrow(\widetilde{C}(\mathcal{H}) \times \widetilde{C}(\mathcal{K}))_{p+q},
$$

commutes with Hochschild boundaries and therefore by composing it with the map $\Omega$ given by (7.1), one obtains the following map

$$
\begin{aligned}
& \left(\left(h_{1} \otimes \cdots \otimes h_{p}\right) \otimes\left(k_{1} \otimes \cdots \otimes k_{q}\right)\right) \\
& \quad \longmapsto \sum_{\sigma} \operatorname{sign}(\sigma)\left(\left(h_{\sigma^{-1}(1)} \otimes 1\right) \otimes \ldots\left(h_{\sigma^{-1}(p)} \otimes 1\right)\right. \\
& \otimes\left(1 \otimes k_{\sigma^{-1}(1)} \otimes \ldots\left(1 \otimes k_{\left.\sigma^{-1}(q)\right)}\right),\right.
\end{aligned}
$$

where $\sigma$ runs through $(p, q)$-shuffles. This map induces a product in Hopf Hochschild homology:

$$
\widetilde{H H}_{p}(\mathcal{H}) \otimes \widetilde{H H}_{q}(\mathcal{K}) \longrightarrow \widetilde{H H}_{p+q}(\mathcal{H} \otimes \mathcal{K}) .
$$


When $\mathcal{H}$ is commutative we can compose the above map with the the multiplication map $\mathcal{H} \otimes \mathcal{H} \longrightarrow \mathcal{H}$ to obtain a product on Hopf Hochschild homology.

Proposition 8.1 Let $\mathcal{H}$ be a commutative Hopf algebra. The map

$$
\times: \widetilde{H H}_{p}(\mathcal{H}) \otimes \widetilde{H H}_{q}(\mathcal{H}) \longrightarrow \widetilde{H H}_{p+q}(\mathcal{H}),
$$

induces a structure of graded commutative algebra on $\widetilde{H H}_{*}(\mathcal{H})$.

Proof Since $\mathcal{H}$ is commutative, the multiplication map $m: \mathcal{H} \otimes \mathcal{H} \longrightarrow \mathcal{H}$ is a Hopf algebra map. Now composing (8.1), for $\mathcal{H}=\mathcal{K}$, with the maps induced by $m$ and $\Omega$, provides us a product map:

$$
\times: \widetilde{C}_{p}(\mathcal{H}) \otimes \widetilde{C}_{q}(\mathcal{H}) \longrightarrow \widetilde{C}_{p+q}(\mathcal{H})
$$

given by

$\left(h_{1} \otimes \cdots \otimes h_{p}\right) \times\left(h_{p+1} \otimes \cdots \otimes h_{p+q}\right)=\sum_{\sigma} \operatorname{sign}(\sigma)\left(h_{\sigma^{-1}(1)} \otimes \cdots \otimes h_{\sigma^{-1}(p+q)}\right)$.

Here $\sigma$ runs over all $(p, q)$-shuffles. Therefore $\widetilde{C}_{*}(\mathcal{H})$ becomes a graded algebra.

Now we define a cup product for Hopf cyclic homology of a commutative Hopf algebra.

Proposition 8.2 Let $\mathcal{H}$ and $\mathcal{K}$ be two Hopf algebras. The following map is a map of (b, B)-mixed complexes:

$$
\star: \operatorname{Tot} \mathcal{B} \widetilde{C}_{p}(\mathcal{H}) \otimes \operatorname{Tot} \mathcal{B} \widetilde{C}_{q}(\mathcal{K}) \longrightarrow \operatorname{Tot} \mathcal{B}(\widetilde{C}(\mathcal{H}) \times \widetilde{C}(\mathcal{K}))_{p+q+1},
$$

given by

$$
\left(x_{p}, x_{p-2}, \ldots\right) \star\left(y_{p}, y_{p-2}, \ldots\right)=\left(B x_{p} \times y_{q}, B x_{p} \times y_{q-2}, \ldots\right) .
$$

Proof It is enough to show:

$$
\begin{aligned}
& b\left(B x_{p} \times y_{q}\right)+B\left(B x_{p} \times y_{q-2}\right) \\
& \quad=B\left(b x_{p}+B x_{p-2}\right) \times y_{q}+(-1)^{p} B x_{p} \times\left(b y_{q}+B y_{q-2}\right),
\end{aligned}
$$

and similarly for the other terms. Let $s h_{i, j}^{\prime}(x, y)=x \times^{\prime} y$. One can verify $B x \times^{\prime}$ $y=0$ and $x \times^{\prime} B y=0$, for all $x$ and $y$ in the normalized complex. The equation $[B, s h]+\left[b, s h^{\prime}\right]=0$ is equivalent to

$$
B(x \times y)-\left(B x \times y+(-1)^{i} x \times B y\right)=-b\left(x \times^{\prime} y\right)+b x \times^{\prime} y+(-1)^{i} x \times^{\prime} b y .
$$

By substituting $B y$ instead of $y$ in the above equation we obtain:

$$
B(x \times B y)=B x \times B y .
$$

Now (8.3) is the consequence of the fact that $[b, s h]=0$. 
Theorem 8.1 The map $\star$ induces the following associative product

$$
\widetilde{H C}_{p}(\mathcal{H}) \otimes \widetilde{H C}_{q}(\mathcal{K}) \longrightarrow \widetilde{H C}_{p+q+1}(\mathcal{H} \otimes \mathcal{K})
$$

in Hopf cyclic homology. If we consider $\widetilde{H C_{n}}(\mathcal{H})$ of degree $n+1$, then the product is graded commutative, i.e.,

$$
x \star y=(-1)^{(p+1)(q+1)}(y \star x),
$$

for $x \in \widetilde{H C}_{p}(\mathcal{H})$ and $y \in \widetilde{H C_{q}}(\mathcal{K})$.

Now we are ready to define a cup product for Hopf cyclic homology of commutative Hopf algebras:

Proposition 8.3 Let $\mathcal{H}$ be a commutative Hopf algebra. The product $\star$ induces a graded commutative algebra structure on Hopf cyclic homology:

$$
\widetilde{H C}_{p}(\mathcal{H}) \otimes \widetilde{H C}_{q}(\mathcal{H}) \longrightarrow \widetilde{H C}_{p+q+1}(\mathcal{H})
$$

Proof This is the consequence of that fact that when $\mathcal{H}$ is commutative, the product map $m$ is a Hopf algebra map.

One can see that

Proposition 8.4 The boundary map $\partial$ in the Künneth long exact sequence (7.2) is the same as the product $\star$ in Hopf cyclic homology,

$$
\partial(x \otimes y)=x \star y,
$$

where $x \in \widetilde{H C}(\mathcal{H})$ and $y \in \widetilde{H C}(\mathcal{K})$.

\section{References}

1. Bauval, A.: Theoréme d'Eilenberg-Zilber en homologie cyclique entiere. Prépublications de Laboratoire Emile Picard, n. 112 (1998)

2. Burghelea, D., Ogle, C.: The Künneth formula in cyclic homology. Math. Z. 193, 527-536 (1986)

3. Connes, A: Cohomologie cyclique et foncteurs $E x t^{n}$. C. R. Acad. Sci. Paris Ser. I Math. 296(23), 953-958 (1983)

4. Connes, A.: Noncommutative differential geometry. Inst. Hautes Études Sci. Publ. Math. 62, 257-360 (1985)

5. Connes, A.: Noncommutative Geometry. Academic Press, San Diego. Available online at http:// alainconnes.org/ (1994)

6. Connes, A., Moscovici, H.: Hopf algebras, cyclic cohomology and the transverse index theorem. Comm. Math. Phys. 198(1), 199-246 (1998)

7. Connes, A., Moscovici, H.: Cyclic cohomology and Hopf algebra symmetry. Lett. Math. Phys. 52(1), $1-28$ (2000)

8. Emmanouil, I.: The Künneth formula in periodic cyclic homology. K-Theory. 10(2), 197-214 (1996)

9. Emmanouil, I.: Mittag-Leffler condition and the vanishing of $\lim ^{1}$. Topology. 35(1), 267-271 (1996)

10. Getzler, E., Jones, J.D.S.: The cyclic homology of crossed product algebras. J. Reine Angew. Math. 445, 161-174 (1993) 
11. Gorokhovsky, A.: Secondary characteristic classes and cyclic cohomology of Hopf algebras. Topology. 41(5), 993-1016 (2002)

12. Hajac, P.M., Khalkhali, M., Rangipour, B., Sommerhäuser, Y.: Stable anti-Yetter-Drinfeld modules. C. R. Acad. Sci. Paris. 338(8), 587-590 (2004)

13. Hajac, P.M., Khalkhali, M., Rangipour, B., Sommerhäuser, Y.: Hopf-cyclic homology and cohomology with coefficients. C. R. Acad. Sci. Paris. 338(9), 667-672 (2004)

14. Kaygun, A.: Products in Hopf-cyclic cohomology. Homol. Homotopy Appl. 10(2), 115-133 (2008)

15. Kaygun, A.: Uniqueness of pairings in Hopf-cyclic cohomology. J. K-Theory. 6(1), 1-21 (2010)

16. Karoubi, M.: Formule de Künneth en homologie cyclique I. (French) [The Künneth formula in cyclic homology. I] C. R. Acad. Sci. Paris Sèr. I Math. 303(12), 527-530 (1986)

17. Karoubi, M.: Formule de Künneth en homologie cyclique II. (French) [The Künneth formula in cyclic homology. II] C. R. Acad. Sci. Paris Sèr. I Math. 303(13), 595-598 (1986)

18. Kassel, C.: Cyclic homology, comodules and mixed complexes. J. Algebra. 107, 195-216 (1987)

19. Kassel, C.: A Künneth formula for the cyclic cohomology of $\mathbb{Z}_{2}$-graded algebras. Math. Ann. 275, 683-699 (1986)

20. Khalkhali, M. Rangipour, B.: A new cyclic module for Hopf algebras. $K$-Theory. 27(2), 111-131 (2002)

21. Khalkhali, M., Rangipour, B.: On the generalized cyclic Eilenberg-Zilber theorem. Can. Math. Bull. 47(1), 33-48 (2004)

22. Khalkhali, M., Rangipour, B.: Cup products in Hopf-cyclic cohomology. C. R. Acad. Sci. Paris. 340(1), 9-14 (2005)

23. Kustermans, J., Rognes, J., Tuset, L.: The Connes-Moscovici approach to cyclic cohomology for compact quantum groups. K-Theory. 26, 101-137 (2002)

24. Loday, J.L.: Cyclic homology. Springer, Berlin/Heidelberg/New York (1992)

25. Rangipour, B.: Cup products in Hopf cyclic cohomology via cyclic modules. Homol. Homotopy Appl. 10(2), 273-286 (2008)

26. Real, P.: Homological perturbation theory and associativity. Homol. Homotopy Appl. 2(5), 51-88 (2000) 